Review

\title{
Role of tyrosine phosphorylation in modulating cancer cell metabolism
}

\author{
Maria Letizia Taddei ${ }^{\mathrm{a}}$, Elisa Pardella ${ }^{\mathrm{b}}$, Erica Pranzini ${ }^{\mathrm{b}}$, Giovanni Raugei ${ }^{\mathrm{b}, *}$, Paolo Paoli $^{\mathrm{b}}$ \\ ${ }^{a}$ Department of Experimental and Clinical Medicine, University of Florence, Viale Morgagni 50, 50134 Florence, Italy \\ b Department of Experimental and Clinical Biomedical Sciences "Mario Serio", Italy
}

\section{A R T I C L E I N F O}

\section{Keywords:}

Tyrosine phosphorylation

Tumor metabolism

Metabolic enzymes

PhosphoSitePlus

\begin{abstract}
A B S T R A C T
In mammalian cells, tyrosine phosphorylation is one of the main mechanisms responsible for regulating signal transduction pathways and key cellular functions. Moreover, recent studies demonstrated that tyrosine phosphorylation influences the activity of some metabolic enzymes, even if it remains to be clarified whether tyrosine phosphorylation can be considered a general mechanism involving most of the metabolic enzymes or only a subset of these. To elucidate this aspect, we conducted a two-step analysis. First, we analyzed literature to identify all the metabolic enzymes whose activity is affected by tyrosine phosphorylation. Second, we crossed these data with those obtained from the PhosphoSitePlus database analysis. Collected information was used to depict an exhaustive map showing the real spread of tyrosine phosphorylation among metabolic enzymes. In summary, data reported in this review highlight that tyrosine phosphorylation is not a sporadic event but a widespread post-translational modification, which is essential to promote the metabolic reprogramming of cancer cells.
\end{abstract}

\section{Introduction}

The term "Warburg metabolism" has been coined in honour of Otto Warburg who described for the first time a peculiar metabolic behaviour of cancer cells about 90 years ago. He highlighted that, in comparison to their healthy counterpart, cancer cells consume much more glucose and release a larger amount of lactate in the extracellular medium, even in the presence of oxygen. To explain this phenomenon, Warburg suggested that cancer cells possess defective/non-functioning mitochondria and have to rely on glycolysis to synthesize metabolic intermediates and generate the ATP for cell growth and proliferation [1].

Starting from these observations, several advances have been made to understand the metabolic reprogramming of cancer cells [2]. Numerous studies brought out that cancer cells possess a plastic metabolism and retain active, although differently functioning, mitochondria. These organelles not only regulate energy metabolism, but also control cell viability by modulating the programmed cell death, are important sources of reactive oxygen species (ROS), and can generate retrograde signals able to affect nuclear gene expression. Moreover, mithocondria produce many essential building block molecules, which are necessary to synthesize nucleotides, lipids, and proteins [3]. Taken together, these findings indicate that mitochondria play a key role in carcinogenesis [4]. This hypothesis was confirmed by further studies showing that any stimulus able to damage the mitochondria of cancer cells impairs their tumorigenic potential [5]. Altogether, these observations suggest that the phenomenon described as "cancer metabolic reprogramming" is not simply characterized by an enhanced glycolytic flux, but it predicts profound changes in almost all known metabolic pathways [6].

In recent years, many efforts have been made to identify the molecular mechanisms that promote such a metabolic deregulation of cancer cells. First of all, we can find germline and somatic mutations of genes encoding key metabolic enzymes [7], overexpression or constitutive activation of oncogenes, and loss of function of tumor suppressor genes such as MYC, RAS, BRAF and TP53 [8].

Among oncogenes, those belonging to the tyrosine kinases (TKs) family represent one of the most important subgroups [9]. To date, more than 30 oncogenic TKs have been identified and characterized in cancer cells [10]. Some of these enzymes are plasma-membrane proteins, while others are located in the cytoplasm, mitochondria or nucleus, and are able to phosphorylate a broad number of targets, including several signaling effectors, transcription factors, enzymes or proteins involved in metabolic pathways [11]. More recently, it has been demonstrated that cancer cells treated with TKs inhibitors reduce glucose consumption and shift toward a respiratory phenotype [12-14]. Finally, it has been elucidated that overexpression or downregulation of protein tyrosine phosphatases (PTPs), the enzymes that physiologically counteract the activity of TKs, contribute to the metabolic rewiring of

\footnotetext{
* Corresponding author at: Department of Experimental and Clinical Biomedical Sciences, University of Florence, Viale Morgagni 50, 50134 Firenze, Italy.

E-mail address: giovanni.raugei@unifi.it (G. Raugei).
} 


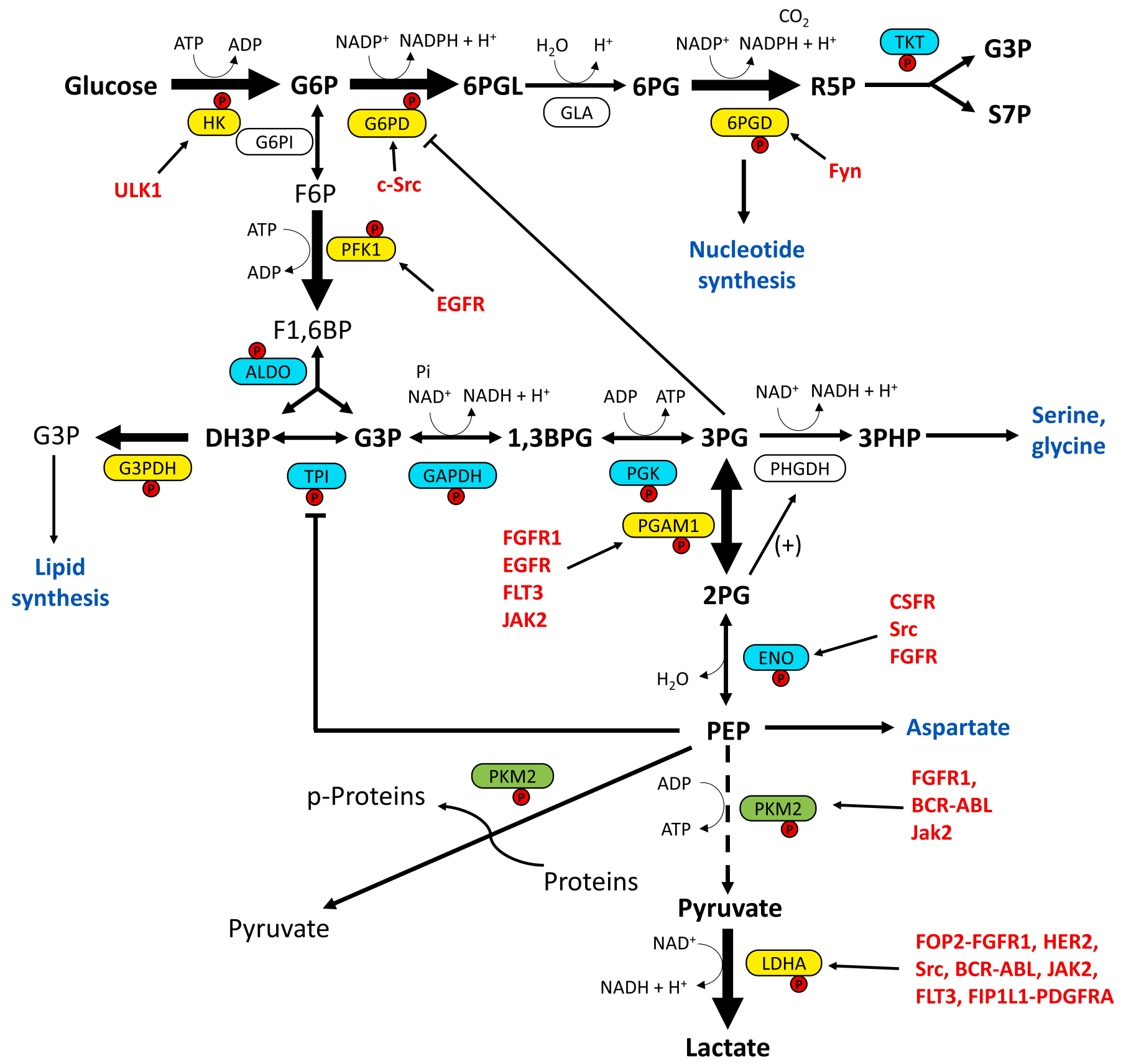

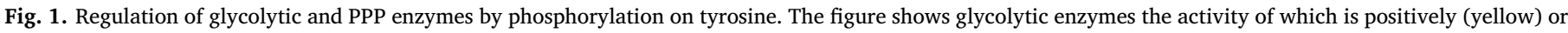

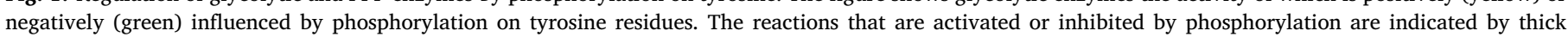

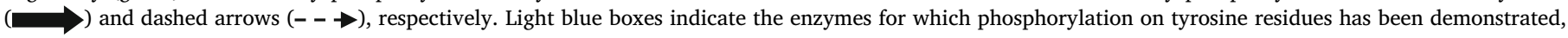

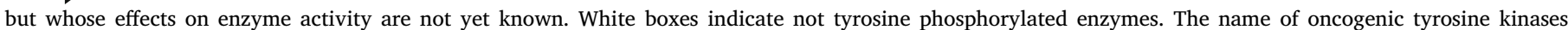

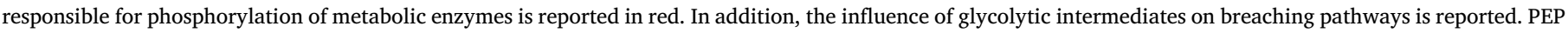

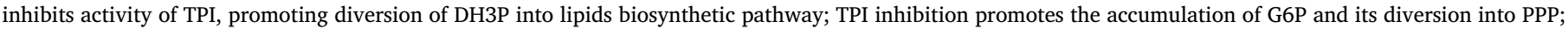

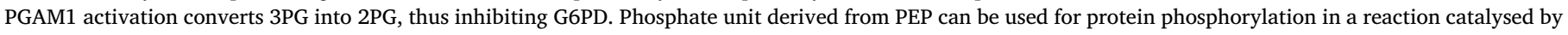
the dimeric form of PKM2. (For interpretation of the references to color in this figure legend, the reader is referred to the web version of this article.)

cancer cells $[15,16]$. Altogether, this evidence suggests that tyrosine phosphorylation could play a more important role than that hitherto attributed in the regulation of the energy metabolism of cancer cells [17].

One aspect that still needs to be clarified is how this post-translational modification can influence the activity of the enzymes. By introducing additional negative charges, phosphorylation can generate attractive or repulsive phenomena which in turn can modify the position of side chains of critical amino acids, such as those present in the catalytic site. Hence, tyrosine phosphorylation can affect catalysis, or modify the affinity of the enzyme for the substrate or cofactors, thereby resulting in its activation or inhibition $[18,19]$. On the other hand, depending on the position and on the number of residues involved, tyrosine phosphorylation can also affect the overall tridimensional structure of the enzymes. This event has often been described for enzymes harbouring tyrosine residues that can be phosphorylated localized at the interface between different domains, or subunits of multimeric enzymes. In all these cases, phosphorylation promotes the assembling or the dissociation of enzymes subunits, inducing the activation or inhibition of the enzymes. That is the case of hexokinase (HK) 
and pyruvate kinase M2 (PKM2), for which it has been reported that the phosphorylation of specific tyrosine residues triggers their dissociation, resulting in the activation of HKs [20], but, conversely, leading to inactivation of PKM2 [21]. Furthermore, it has been described that tyrosine phosphorylation can also affect the localization of metabolic enzymes inside the cell or can modulate their interaction with effectors molecules, thereby boosting or dampening specific metabolic pathways $[22,23]$. Thus, given the range of possible consequences related to tyrosine residues phosphorylation, understanding the final effect of this post-translational modification on metabolic enzymes that harbour multiple phosphorylation sites targeted by different oncogenic TKs results to be very complicated. In these cases, it is possible to observe a differential impact of each tyrosine residue on the overall enzyme activity, as well as synergistic or antagonistic effects.

Despite numerous evidence proves the importance of tyrosine phosphorylation in the regulation of cancer cell metabolism [24], the real extension of this phenomenon among metabolic enzymes is still largely unknown. This review aims to summarize all the findings available so far correlating cancer metabolic adaptations with differential tyrosine phosphorylation in order to clarify the real impact of this post-translational modification on metabolic enzyme activity. Data obtained by a systematic study of literature and PhosphoSitePlus database analysis [25] were used to build up a map of all the phosphorylated metabolic enzymes, providing, for the first time, a clear overview of the real impact of tyrosine phosphorylation on metabolic enzyme activity in cancer cells.

\section{Enzymes with phosphotyrosine-regulated activity}

\subsection{Glycolysis}

It is well known that cancer cells primarily use glycolysis as an anabolic pathway to sustain cell growth and proliferation [26]. This requires the increase of the glycolytic flux and the activation of corresponding regulatory mechanisms, inducing the redirection of a large part of metabolic intermediates into biosynthetic pathways. Accordingly, almost all the glycolytic enzymes, including HK, glucose 6phosphate isomerase (G6PI), phosphofruttokinase-1 (PFK1), fructose1,6-bisphosphatase 1 (FBPase), aldolase (ALDO), triosephosphate isomerase (TPI), glyceraldehyde 3-phosphate dehydrogenase (GAPDH), phosphoglycerate kinase (PGK), phosphoglycerate mutase (PGAM), and $\alpha$-enolase (ENO), are activated by phosphorylation on tyrosine residues, thereby confirming that oncogenic TKs exert a capillary control on enzymes involved in this pathway (Fig. 1, Supplementary Table S1).

\subsubsection{Hexokinase (HK)}

$\mathrm{HK}$ is the first and rate-limiting enzyme of glycolysis. It catalyses the irreversible phosphorylation of glucose to glucose-6-phosphate (G6P), using ATP as the phosphate donor. HK is localized in the cytosol and four different isozymes of HK (namely, HK-I, II, III, IV), catalysing the same reaction, have been described. HK2 expression is a specific feature of several tumors, even those arising in tissues where normally only HK1 is expressed. The importance of HK2 in supporting tumor progression is demonstrated by the promising results obtained thanks to the application of targeted therapies developed specifically against HK2 [27].

In a recent study, Zhang and co-authors demonstrated that HK1 and HK2 isoforms are phosphorylated by c-Src on Y732 and Y686 residues, respectively. Tyrosine phosphorylation affects HK activity through two mechanisms: it improves the affinity of $\mathrm{HK}$ for its substrate and it promotes the dissociation of the homo-dimeric form of HK into the monomeric form that has a higher catalytic activity. Thus, Src, by phosphorylating both HK isoforms on tyrosine residues, contributes to drive glucose into the glycolytic pathway, promoting cell proliferation [20].

\subsubsection{Phosphofruttokinase (PFK)}

PFK-1 transfers a phosphate group from ATP to fructose-6-phosphate (F6P), converting it into fructose-1,6-bisphosphate (F-1,6- $\mathrm{P}_{2}$ ). This reaction is essentially irreversible under intracellular conditions. PFK-1 activity is strongly increased in cancer cell lines and primary tumor tissues responding to the high requirement of neoplastic cells for increased glycolysis $[28,29]$. This hypothesis is also supported by the evidence that the oncogenes Ras and Src activate PFK in immortalized cells [30].

Lee and co-authors demonstrated that, in glioblastoma, EGFR binds and phosphorylates the platelet isoform of PFK1, namely PFKP, on Y64 residue. Consequently, tyrosine-phosphorylated PFK can bind the $\mathrm{N}$ terminal SH2 domain of $\mathrm{p} 85 \alpha$, promoting the activation of the phosphoinositide 3-kinase (PI3K). The subsequent PI3K-dependent AKT activation results in enhanced phosphorylation of PFK2, which catalyses the synthesis of fructose-2,6-bisphosphate $\left(\mathrm{F}-2,6-\mathrm{P}_{2}\right)$, a potent allosteric activator of PFK1 [22]. Meanwhile, it has been recently demonstrated that AKT can also phosphorylate $\beta$-catenin, promoting its nuclear translocation and transactivation, thus resulting in enhanced expression of its downstream genes, such as CCND1 and MYC [31]. This event is essential to trigger the expression of GLUT1, PKM2, and the A isoform of lactate dehydrogenase (LDHA), thereby promoting glycolysis, proliferation and migration in cancer cells [31].

\subsubsection{Triosephosphate isomerase (TPI)}

TPI is the enzyme that reversibly converts dihydroxyacetone phosphate (DHAP) into glyceraldehyde 3-phosphate (G3P) and it is known that serine/threonine phosphorylation by the cyclin A/Cdk2 complex promotes its inactivation [32]. Moreover, it has been recently demonstrated that TPI is tyrosine phosphorylated in A375 melanoma cells and a specific tyrosine phosphatase, the low molecular weight protein tyrosine phosphatase (LMW-PTP), is responsible for its dephosphorylation [16]. LMW-PTP is overexpressed in many aggressive human tumors [33]. Interestingly, its overexpression leads to enhanced oxidative phosphorylation (OXPHOS) metabolism. On the contrary, LMW-PTP silencing stimulates glucose uptake, lactate release, and promotes cancer cell proliferation, while decreases oxygen consumption, cell migration and invasiveness [16].

\subsubsection{Glyceraldehyde 3-phosphate dehydrogenase (GAPDH)}

GAPDH is the sixth enzyme of glycolysis and it participates in the energy-releasing phase of this metabolic pathway. It plays a fundamental role in both glycolysis and gluconeogenesis by reversibly catalysing the oxidation and the phosphorylation of G3P in 1,3Bisphospshoglycerate (1,3-BPG), using $\mathrm{NAD}^{+}$as co-substrate. In this reaction, the aldehydic group of G3P is initially oxidized to a carboxylic acid by $\mathrm{NAD}^{+}$, which in turn is converted in its reduced form NADH; then, the carboxylic group reacts with orthophosphate $\left(\mathrm{P}_{\mathrm{i}}\right)$, releasing the product 1,3-BPG, a highly-energy phosphate compound.

Recent evidence indicates that GAPDH is involved in different functions not specifically related to its role in glycolytic metabolism, such as regulation of cell death, autophagy, DNA repair, and RNA export [34]. The elucidated correlation between different subcellular compartmentalization of GAPDH and the induction of the autophagic process in cancer cells is noteworthy. These additional roles of GAPDH open the possibility of considering it as an interesting pharmacological target [35]. Another non-metabolic role of GAPDH is the promotion of Rab2 mediated vesicle fusion. Interestingly, it has been reported that GAPDH is phosphorylated on Y41 by Src. Even if there is no evidence that this specific modification influences GAPDH enzymatic activity in glycolysis, it seems to impact on its role in inducing vesicle fusion [36]. A GAPDH mutant defective in Src-dependent tyrosine phosphorylation (GAPDH Y41F) impedes Rab2-mediated events [37]. Moreover, GAPDH is also phosphorylated on Y94 residue: indeed, evidence shows that such modification alters the molecular dynamic parameters of intranuclear GAPDH, probably by inhibiting its interactions with not yet 
identified nuclear biomolecules [38]. Recently, it has been demonstrated that LMW-PTP is able to interact with and dephosphorylate tyrosine-phosphorylated GAPDH [16]. However, the identity of the dephosphorylated tyrosine residue remains to be established.

\subsubsection{Phosphoglycerate kinase (PGK)}

PGK reversibly transfers the phosphate group of the highly-energy compound 1,3-BPG, generated in the previous reaction catalysed by GAPDH, to ADP in order to produce ATP and 3-phosphoglycerate (3PG). As nicely summarized in a recent review from He and co-authors, PGK1 plays an important role in mediating cancer progression and drug resistance, thanks to both its role in glycolysis and its kinase activity towards different substrates [39].

Shetty and co-authors reported that the binding of urokinase-type plasminogen activator (UPA) to its receptor (UPAR) promotes phosphorylation of the Y76 residue of PGK. Moreover, they also demonstrated that tyrosine phosphorylation of PGK enhances UPAR mRNA stabilization, thereby stimulating receptor synthesis [40]. To date, no evidence is available about the effect of Y76 phosphorylation on the enzyme activity but mutational studies revealed that inhibition of tyrosine phosphorylation increases PGK binding to uPAR mRNA and attenuates uPA-induced UPAR expression [40]. Interestingly, it has been shown that, upon overexpression, UPAR assembles with and activates $\alpha 5 \beta 1$-integrin and EGFR, thereby favouring the activation of PI3K/ $\mathrm{pAKT} / \mathrm{mTOR} / \mathrm{HIF}-1 \alpha$ signaling pathway [41]. HIF-1 $\alpha$ stabilization promotes the expression of glycolytic genes, thereby sustaining the Warburg effect. In conclusion, this evidence suggests that phosphorylation at Y76 residue of PGK indirectly promotes the Warburg effect in melanoma cells.

\subsubsection{Phosphoglycerate mutase (PGAM1)}

PGAM1 is the eighth enzyme of the glycolysis, that catalyses the reversible transfer of the phosphate group between C-2 and C-3 of glycerate, thereby converting 3-PG into 2-phosphoglycerate (2-PG). The reaction consists in two different steps: initially, the phosphorylated form of PGAM1 transfers its phosphate group from the histidine residue (H11) to the C-2 of 3-PG, converting it into 2,3-bisphosphoglycerate (2,3-BPG); then, the C-3 phosphate group of 2,3-BPG is released back to the histidine residue of the enzyme, ultimately producing 2-PG. Recent papers indicate that PGAM1 may promote the progression of the most aggressive forms of several different types of tumor. In fact, PGAM1 overexpression correlates with cell proliferation and survival. The cancer-specific role of PGAM1 and the possible application of specific inhibitors for targeted therapy are well described in a recent review from Sharif and co-authors [42].

In the context of tyrosine phosphorylation, it is attractive the paper form Hitosugi and co-authors, who reported that PGAM1 is a target of FGFR and other kinases, resulting in the phosphorylation of multiple tyrosine sites, including Y26, Y92, Y119, and Y133 residues. However, among all, only the Y26 phosphorylation influences the enzyme activity. In particular, they demonstrated that Y26 phosphorylation causes displacement of the E19 residue side chain away from the active site of the enzyme, thus allowing the binding of 2,3-BPG cofactor, and resulting in the phosphorylation of histidine 11 (H11) of PGAM1. The active site of PGAM1, harbouring both Y26 and H11 residues in the phosphorylated form, results more accessible to the substrate 3-PG. In conclusion, Y26 phosphorylation promotes $\mathrm{H} 11$ phosphorylation and enhances substrate binding, thereby contributing to PGAM1 activation [19]. Moreover, in vitro studies on H1229 cancer cells and in vivo analysis of xenograft nude mice revealed the importance of Y26 phosphorylation (which is a common modification in several cancer cell types) for glycolysis, RNA biosynthesis, lipogenesis and in vivo tumor growth [19]. Interestingly, it has been reported that the two glycolytic intermediates, namely 3-PG and 2-PG, regulate the activity of extraglycolytic enzymes. Indeed, 3-PG inhibits 6-phosphogluconate dehydrogenase (6-PGD) of the oxidative branch of pentose phosphate pathway (PPP), while 2-PG activates phosphoglycerate dehydrogenase (PHGDH), the first enzyme of the serine biosynthetic pathway [43]. Therefore, it is reasonable to think that oncogenic TKs, regulating glycolytic enzymes, that display multiple phosphorylable tyrosine residues, can modulate the functions of extra-glycolytic enzymes involved in biosynthetic pathways, by changing the intracellular concentration of regulatory metabolites [44]. Unfortunately, only in a few cases this hypothesis is confirmed by mutational studies that further substantiate that tyrosine phosphorylation is essential to promote the Warburg effect and sustain cancer progression [19].

\subsection{7. $\alpha$-enolase (ENO1)}

ENO1 converts 2-PG in phosphoenolpyruvate (PEP), by catalysing the removal of a water molecule from 2-PG. Interestingly, in colorectal cancer, ENO1 induces tumorigenesis and metastasis, acting on the AMPK/mTOR pathway [45]. Moreover, the involvement of ENO1 in promoting a more aggressive phenotype and in favouring the development of chemoresistance has been demonstrated also in gastric cancer [46]. A recent study performed on A375 and PC3 cells demonstrated that ENO1 is one of the glycolytic enzymes whose phosphorylation increases after the silencing of LMW-PTP [16]. Importantly, in these cells tyrosine phosphorylation of ENO1 is required to promote Warburg metabolism thus prompting cell proliferation [16].

Moreover, Cooper and co-workers showed that in fibroblasts transfected with ROUS sarcoma virus, which encodes for $\mathrm{pp} 60^{\mathrm{v} \text {-src }}$, the ENO1 resulted phosphorylated on a tyrosine residue. To date, the identity neither of the tyrosine residue or its effect on the catalytic enzyme activity is known [47].

\subsubsection{Pyruvate kinase M2 (PKM2)}

PKM2 is the muscle isozyme of the pyruvate kinase, the last enzyme of the glycolytic pathway, transferring the phosphate group from PEP to ADP in order to produce pyruvate and ATP. Several findings indicate that in tumor cells PKM2 is generally upregulated and that it mainly exists as dimer, with a lower catalytic activity. This adaptation promotes the anabolic synthesis through the PPP pathway, thus supporting cancer cell proliferation and growth. In addition, it has been assessed that also the protein kinase activity of this enzyme contributes to tumorigenesis [48]. The therapeutic relevance of PKM2 inhibitors as anticancer drugs has been recently suggested, also in light of the known relationship between PKM2 expression and drug resistance in tumor cells [49].

Phospho-proteomic analysis of extracts derived from different cancer cells revealed that several tyrosine residues of PKM2 may be regulated by opposite actions of protein TKs and phosphatases, such as Y83, Y105, Y148, Y175, Y370, and Y390 [15,16,21]. Nevertheless, mutational analysis revealed that only the phosphorylation of Y105 and Y148 residues impairs enzyme activity $[21,50]$. Y105 phosphorylation is triggered by various tyrosine kinases, such as FGFR1, BCR-ABL and Jak2 [21]. From a mechanistic point of view, it has been demonstrated that Y105 phosphorylation obstacles the binding of F-1,6- $\mathrm{P}_{2}$ to PKM2, promoting the dissociation of PKM2 tetramer into the inactive dimeric form [51]. As a consequence, PEP and other glycolytic intermediates are redirected into anabolic pathways, while OXPHOS is inhibited [52]. Conversely, dephosphorylation of Y105 residue by TKs inhibitors increases PKM2 activity, promotes pyruvate production and boost OXPHOS [21]. Furthermore, protein tyrosine phosphatase 1B (PTP1B) and LMW-PTP are able to dephosphorylate PKM2 [15,16]. Indeed, substrate trapping and mutagenesis experiments revealed that PTP1B contributes to the dephosphorylation of PKM2 Y105 residue [15]. Moreover, a recent study conducted on A375 and PC3 cells demonstrated that LMWPTP silencing triggers increased phosphorylation of PKM2 [16].

It is important to underline that PKM2 is the only glycolytic enzyme to be inhibited by phosphorylation on tyrosine [51]. PKM2 phosphorylation/inhibition allows the accumulation of glycolytic intermediates and their rewiring in biosynthetic pathways, a condition described as 
"metabolic jam", which is essential for sustaining cancer cell growth [51]. Moreover, this phenomenon allows some metabolites to reach a critical concentration and, thus, to act as "regulatory factors", controlling the activity of the upstream metabolic enzymes [53]. For example, PEP inhibits the activity of TPI, thereby blocking the conversion of DHAP into G3P. As a consequence, DHAP can be used to synthesize glycerol, a scaffold molecule required for lipid synthesis [54]. At the same time, TPI inhibition contributes to the accumulation of G6P which, in turn, can be diverted into PPP, thereby enhancing the synthesis of NADPH and ribose, improving the production of nucleotides [55]. On the other hand, PEP can be used as a phosphate donor by PKM2, which is stabilized in the dimeric form by tyrosine phosphorylation and can migrate into the nucleus, where it acts as a TK and phosphorylates different targets. By this mechanism, PKM2 contributes to enhance the transcription of proteins and enzymes that finally sustain the Warburg metabolism [56].

\subsubsection{Lactate dehydrogenase ( $L D H)$}

It is well known that in cancer cells a large part of pyruvate is converted into lactate and then released in the extracellular medium [57]. This phenomenon can be explained by the fact that in cancer cells LDHA, the enzyme that catalyses the conversion of pyruvate into lactate, is usually more active than in normal cells [58]. Specifically, it has been shown that LDHA, on one hand, is aberrantly over-expressed in different kinds of cancer and, on the other, displays an elevated rate of tyrosine phosphorylation, which leads to increased enzyme activity. Both conditions are associated with malignant progression [57] and sustain the Warburg effect in cancer cells [59]. Besides lactate synthesis, LDHA also converts cytosolic NADH into $\mathrm{NAD}^{+}$, which is necessary to support the activity of GAPDH and other enzymes involved in biosynthetic pathways, destined to amino acids, lipids, and nucleotides production [60].

LDHA activity is strictly regulated by several oncogenic TKs, including FOP2-FGFR1, HER2, Src, BCR-ABL, the Val617Phe mutant of JAK2, FLT3-internal tandem duplication [ITD] mutant, and FIP1L1PDGFRA kinase [18,59]. Several tyrosine residues can be phosphorylated in LDHA, but only the phosphorylation of Y10 and Y83 residues has been demonstrated to stimulate LDHA activity [18]. Indeed, it has been established that Y10 phosphorylation promotes the assembling of the active (tetrameric) LDHA form, while Y83 phosphorylation enhances the affinity of NADH for the enzyme active site [18]. Moreover, Liu and co-workers recently demonstrated that phosphorylation of the four tyrosine residues (Y10, Y83, Y172, Y239) of LDHA by FGFR1 extended the half-life of the enzyme in prostate cancer [61] (Fig. 2).

Interestingly, data obtained from the PhosphoSitePlus database confirmed that the Y239 residue of LDHA is frequently phosphorylated in cancer cells, even if it is still not described whether this residue has a role in regulating LDHA activity [62].

Furthermore, it has been reported that some cancer cells overexpress also LDHB, the LDH isoform responsible for the conversion of lactate to pyruvate. In these types of cancer, LDHB promotes the utilization of lactate as a carbon source to sustain oxidative metabolism and gluconeogenesis [63]. However, data obtained from PhosphositePlus database show that in the majority of cancer cells this isoform is normally phosphorylated on Y94 and Y240 residues but not on Y10, the residue that mediates the formation of the active LDHA tetramer. A recent study reports that Aurora-A-mediated phosphorylation of LDHB on Ser162 residue improves the affinity of LDHB for pyruvate, thereby leading LDHB to convert pyruvate and NADH into lactate and $\mathrm{NAD}^{+}$, respectively [64]. Altogether, these data suggest that the activity of LDH isoforms is controlled by different post-translational modifications and phosphorylation of Y10 residue represents the mechanism by which oncogenic TKs can selectively activate LDHA. Accordingly, it has been demonstrated that the tumor suppressor cyclin G2 interacts with LDHA preventing the phosphorylation of Y10 residue, thus resulting in the reduction of glycolysis and cancer cell proliferation [65].

\subsection{Pentose Phosphate Pathway (PPP)}

PPP plays an important role in cancer progression by providing precursors for nucleic acid biosynthesis and NADPH for the maintenance of redox balance [66]. In the following sections, PPP enzymes and their phosphorylation on tyrosine residues will be described (Fig. 1, Supplementary Table S2).

\subsubsection{Glucose-6-phosphate dehydrogenase (G6PD)}

G6PD catalyses the first reaction of PPP and oxidizes G6P to 6phosphoglucono- $\delta$-lactone. $\mathrm{NADP}^{+}$acts as the electron acceptor in this reaction and thus it is converted to NADPH. It has been demonstrated that in many types of cancers the over-expression of G6PD enhances cell proliferation and promotes cell survival thanks to its action in modulating redox signaling [67].

The only study available about the regulation of G6PD activity through tyrosine phosphorylation was performed by Pan and coworkers. By investigating whether G6PD may be regulated by tyrosine phosphorylation in endothelial cells in response to VEGF stimulation, they found that tyrosine phosphorylation induced by Src leads to the translocation of the enzyme to the plasma membrane and to the increase of its enzymatic activity. Y428 and Y507 are very likely the two amino acids that undergo phosphorylation [23].

\subsubsection{6-phosphogluconate dehydrogenase (6PGD)}

6-phosphoglucono- $\delta$-lactone is converted in ribulose-5-phosphate by 6PGD, that catalyses an oxidative decarboxylation reaction. It is noteworthy that this step of the PPP pathway produces another molecule of NADPH.

In a very recent paper [68] it has been shown that 6PGD is activated upon tyrosine phosphorylation. EGFR activation elicits the Y481 phosphorylation, driven by the Src family kinase Fyn upon EGF stimulation. Y481 is located very close to the $\mathrm{NADP}^{+}$cofactor-binding site and once phosphorylated it is able to improve the catalytic efficiency of the enzyme, increasing both binding affinity to $\mathrm{NADP}^{+}$and turnover rate of 6PGD. This modulation results in higher NADPH and ribose-5phosphate production, favouring tumor growth. As assessed by the analysis of a large set of tumor samples, phosphorylation of Y481 is associated with Fyn expression and correlates with higher malignancy and a worse prognosis in human glioblastoma.

\subsubsection{Transketolase (TKT)}

TKT plays a fundamental role in the PPP pathway, by allowing the interconversion of the PPP intermediates to ultimately synthesize NADPH and produce $\mathrm{CO}_{2}$ from G6P. Specifically, TKT catalyses the transfer of a fragment with two carbon atoms from the ketose xylulose5 -phosphate to the aldose ribulose-5-phosphate, leading to glyceraldehyde-3-phosphate and sedoeptulose-7-phosphate.

Although it is known that TKT is phosphorylated on tyrosine residues $[23,68]$, to date, no data are available about the implications of this post-translational modification on its activity.

Nevertheless, recent studies revealed the key role of TKT in promoting the Warburg effect [69]. In cancer cells, TKT activity promotes glucose uptake, recycling metabolic intermediates, such as G6P and F6P, into the oxidative harm of PPP [69]. By this mechanism, TKT contributes to maintain low levels of F-1,6- $\mathrm{P}_{2}$ and 3-PG, two metabolites that have a regulatory role, acting the first as a potent PKM2 activator, and the latter as a G6PD inhibitor. Meanwhile, by sustaining the flux of metabolites into the oxidative branch of PPP, TKT promotes the synthesis of NADPH, which is used by isocitrate dehydrogenase (IDH) to promote reductive carboxylation of $\alpha$-ketoglutarate ( $\alpha-\mathrm{KG}$ ), thereby regulating intracellular ROS [70]. Indeed, TKT silencing correlates with increased intracellular levels of $\alpha$-KG, which, in the absence of NADPH, accumulates into the cells, thereby promoting hydroxylation and degradation of HIF-1 $\alpha$ and the shift of metabolism toward OXPHOS [71]. Finally, TKT knockdown causes a decrease of ADP, ATP, and GTP 


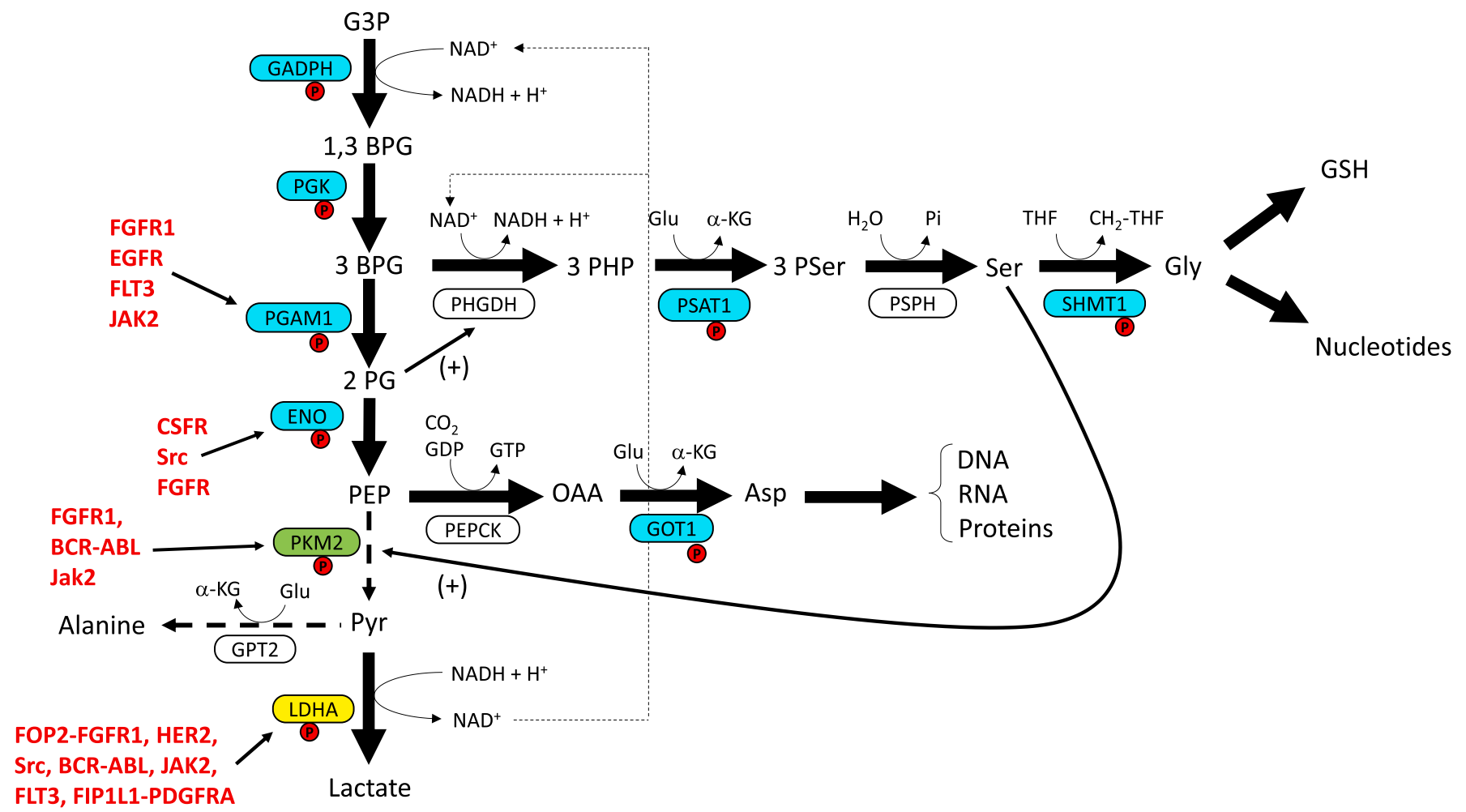

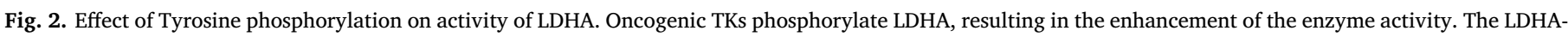

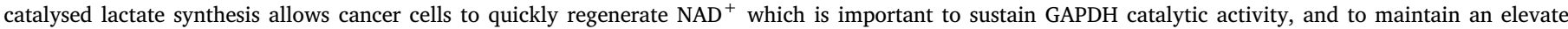

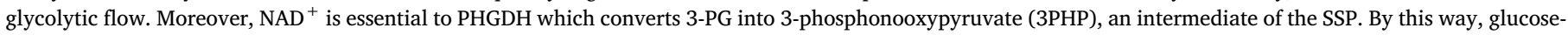

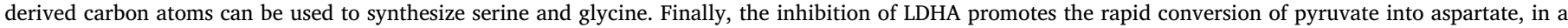

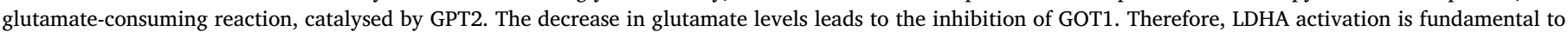

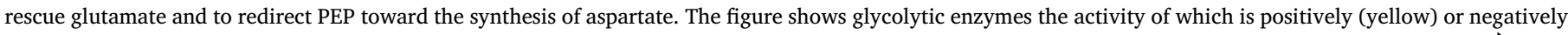

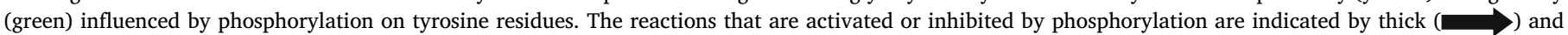

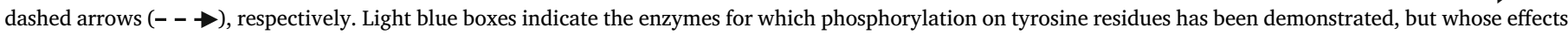

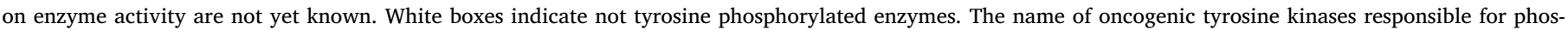

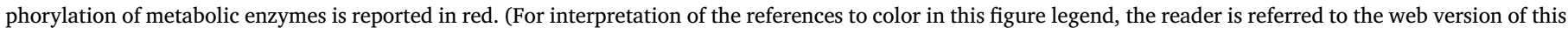
article.)

intracellular levels, suggesting that TKT also regulates the synthesis of purine nucleotides [69]. Confirming its key role in promoting the switch toward a glycolytic metabolism, TKT is overexpressed in the majority of aggressive glycolytic tumors [71]. Interestingly, PhosphoSitePlus database analysis underlined that TKT is highly phosphorylated in cancer cells. Although we do not know which is the effect of phosphorylation of Y275 residue, considering all the above observations, we can expect that phosphorylation of this enzyme may be functional to modulate its activity in cancer cells. Further analyses will be necessary to confirm this hypothesis.

\subsection{NAD and NADP synthesis}

Reductive carboxylation of $\alpha$-KG requires NADPH, which can be directly generated into mitochondria by nicotinamide nucleotide transhydrogenase (NNT), an enzyme that shows multiple phosphorylated tyrosine residues according to data obtained from PhosphoSitePlus database. This enzyme catalyses the transfer of reducing equivalents from NADH to NADPH. By modulating NNT activity, it is possible to control glucose and glutamine metabolism, which can be used as alternative sources of $\alpha$-KG [72,73]. This finding suggests that cancer cells expressing low NNT levels are not able to compensate for NADPH production by PPP, and that NNT activity is essential to refill the intracellular NADPH supply [73]. A recent study focused the attention on the involvement of NNT in gastric cancer, demonstrating that NNT overexpression correlates with increased cell survival, thanks to the antioxidant activity of this enzyme. This hypothesis was also confirmed by knockdown experiments which lead to higher ROS levels and to an increase in apoptotic cell death. Finally, in vivo experiments confirmed a role for NNT also in inducing metastatic dissemination of gastric cancer [74].

Based on all these evidence, it is possible to suppose that, besides its overexpression, the phosphorylation of NNT tyrosine residues modulates its activity and maintains the metabolic flexibility of cancer cells, allowing them to enhance the flux of glutamine-derived carbons into Krebs cycle under glucose deprivation, or, conversely, to increase glucose metabolism when the availability of glutamine decreases.

Many oncogenes enhance the expression of the enzymes involved in the nicotinamide adenine dinucleotide synthesis pathways, thus meeting the increased demand of $\mathrm{NAD}^{+}$in proliferating cells [75]. Data reported in this review show that both nicotinate phosphoribosyltransferase, (NAPRT1) and nicotinamide phosphoribosyltransferase (NAMPT) are phosphorylated on tyrosine residues in cancer cells, suggesting that the activity of these enzymes is under the control of oncogenic TKs. The $\mathrm{NAD}^{+}$coenzyme is required to sustain the activity of a plethora of metabolic and non-metabolic enzymes, such as sirtuins [76]. These enzymes are generally activated when $\mathrm{NAD}^{+}$level increases, resulting in the inhibition of HIF- $1 \alpha$ transcriptional activity, in the reduction of glucose metabolism and in the modulation of the Krebs cycle [77]. Although so far the impact of tyrosine phosphorylation on the activity of NAPTR1 and NAMPT remains to be elucidated, it is conceivable that this phenomenon could be another mechanism by 
which oncogenic TKs are able to control fluxes of metabolites and energy metabolism of cancer cells (Supplementary Table S3).

\subsection{Krebs cycle}

One of the most important consequences of cancer metabolic reprogramming is the strong reorganization of mitochondrial metabolism and the switch from a catabolic to a biosynthetic use of the Krebs cycle [6]. The effect of mutations of different TCA cycle enzymes on carcinogenesis is quite well documented. These mutations cause abnormal accumulation of oncometabolites produced by the TCA cycle, stimulating oncogenesis and cancer progression. In a recent review from Sajnani and co-authors, the importance of TCA cycle in cancer progression is well summarized, pointing particular attention to the altered activity of aconitase and IDH described in several types of cancer [78]. However, data reported in this review show that all the enzymes involved in the Krebs cycle are phosphorylated in tyrosine residues by oncogenic TKs (Figs. 3-4 and Supplementary Table S4). This finding renfoces the hypothesis that tyrosine phosphorylation has a key role in modulating the Krebs cycle.

\subsubsection{Pyruvate dehydrogenase complex (PDC)}

In mammalian cells, the mitochondrial PDC is responsible for the conversion of pyruvate into acetyl coenzyme A (Acetyl-CoA), fuelling the glucose-derived carbon atoms into the Krebs cycle. One of the most important steps to achieve the shift to a biosynthetic use of the Krebs cycle in cancer cells is to block the conversion of pyruvate into AcetylCoA by inhibiting the activity of PDC. Indeed, most of the pyruvate produced by cancer cells is not metabolized by mitochondria because PDK, a TK in its turn activated by oncogenic TKs, phosphorylates on tyrosine residues PDC thereby inactivating it $[79,80]$.
PDC includes several enzymes, such as the pyruvate dehydrogenase (PDH1, E1), the dihydrolipoyl transacetylase (E2), the dihydrolipoamide dehydrogenase (E3), the pyruvate dehydrogenase kinase (PDHK1) and the pyruvate dehydrogenase phosphatase (PDP1). Phosphorylation of PDC by PDHK1 leads to PDC inactivation, whereas dephosphorylation by PDP1 re-establishes PDC activity. In differentiated cells, PDH1 associates with PDP1 and with $\mathrm{NAD}^{+}$-dependent deacetylase sirtuin-3 (SIRT3), which maintains PDH1 in the deacetylated (active) form [81].

Moreover, it has been demonstrated that different TKs, including EGFR, FGFR1, FLT3 and JAK2, phosphorylate PDP1 on the Y381 residue. Phosphorylation of this residue leads to the dissociation of SIRT3 from the PDH1-PDP1 complex, thereby favouring the recruitment of acetyl-CoA acetyltransferase (ACAT1). ACAT1 acetylates both PDH1 and PDP1 on lysine residues, promoting their dissociation. Subsequently, acetylated PDH1 recruits PDHK1 that, in turn, phosphorylates PDH1 on Ser293 residue, inhibiting its activity [79]. Finally, it is important to highlight that Y381 is not the only PDP1 tyrosine residue phosphorylated by TKs in cancer cells. It has been recently demonstrated that also phosphorylation of Y94 residue contributes to the inhibition of PDC complex activity, promoting the Warburg effect in cancer cells [80].

Further studies revealed that both PDH1 and PDHK1 are phosphorylated by oncogenic TKs $[81,82]$. In cancer cells several kinases, including FGFR1, EGFR, BCR-ABL, JAK2, EOL1, KG-1a, Molm14, Mo91 and FLT3, catalyse PDH1 phosphorylation on Y301 residue, blocking the binding of the pyruvate to the enzyme. These findings suggest that Y301 phosphorylation inhibits OXPHOS and stimulates glycolysis, providing a metabolic advantage to cell proliferation under hypoxia [81]. On the other hand, it has been reported that mitochondrial-localized kinases, such as FGFR1, FLT3, BCR-ABL and JAK2, trigger

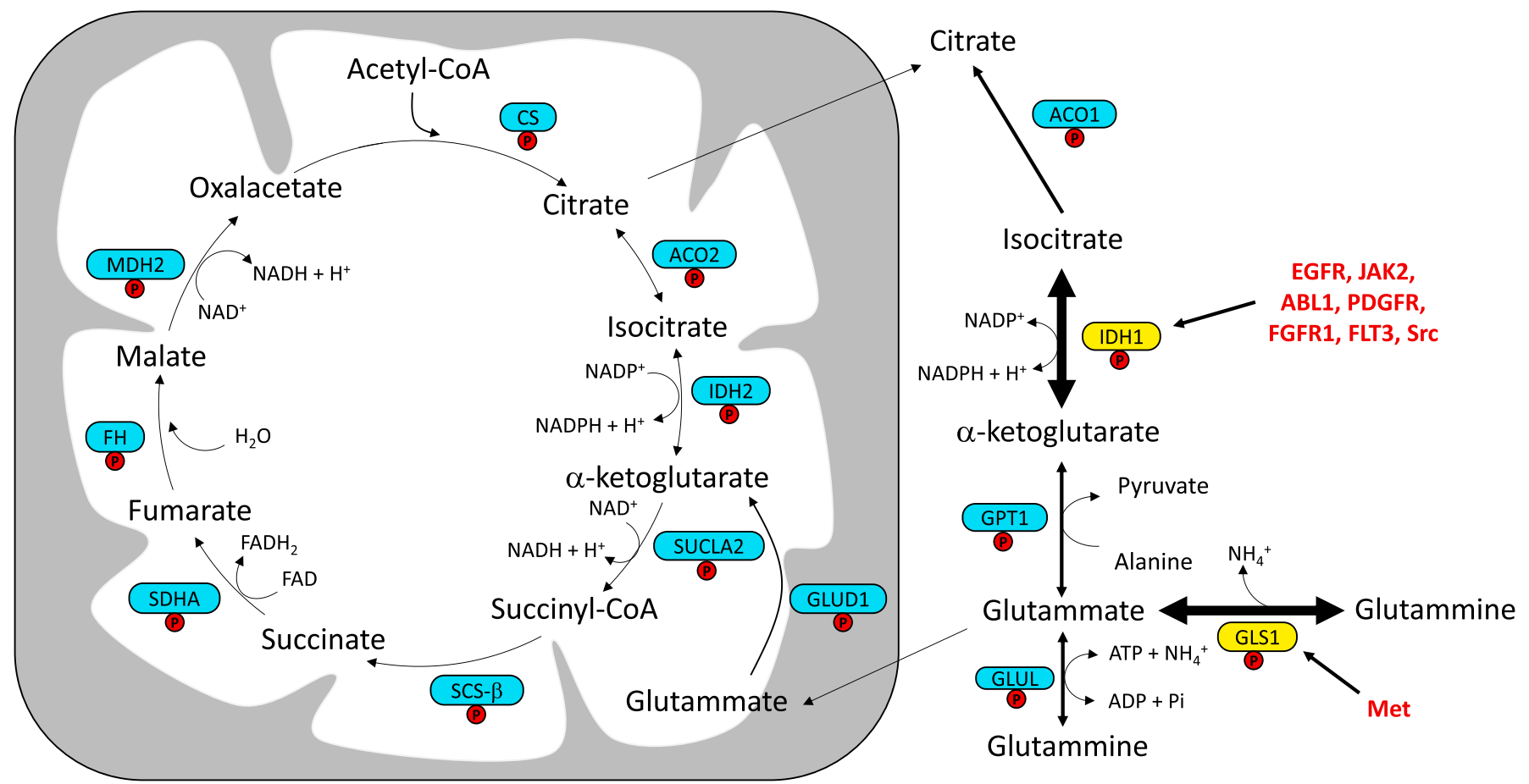

Fig. 3. Regulation of Krebs cycle by phosphorylation on tyrosine. All the enzymes of the Krebs cycle result to be phosphorylated on tyrosine residues. In particular, IDH1 phosphorylation enhances its activity promoting the synthesis of $\alpha$-KG from isocitrate favouring cancer progression through different mechanisms. Tyrosine phosphorylation enhances GLS1 activity thereby increasing $\alpha$-KG production and feeding the Krebs cycle. The figure shows glycolytic enzymes the activity of which is positively (yellow) or negatively (green) influenced by phosphorylation on tyrosine residues. The reactions that are activated or inhibited by phosphorylation are indicated by thick $(\longrightarrow$ ) and dashed arrows $(-\rightarrow$ ), respectively. Light blue boxes indicate the enzymes for which phosphorylation on tyrosine residues has been demonstrated, but whose effects on enzyme activity are not yet known. White boxes indicate not tyrosine phosphorylated enzymes. The name of oncogenic tyrosine kinases responsible for phosphorylation of metabolic enzymes is reported in red. (For interpretation of the references to color in this figure legend, the reader is referred to the web version of this article.) 


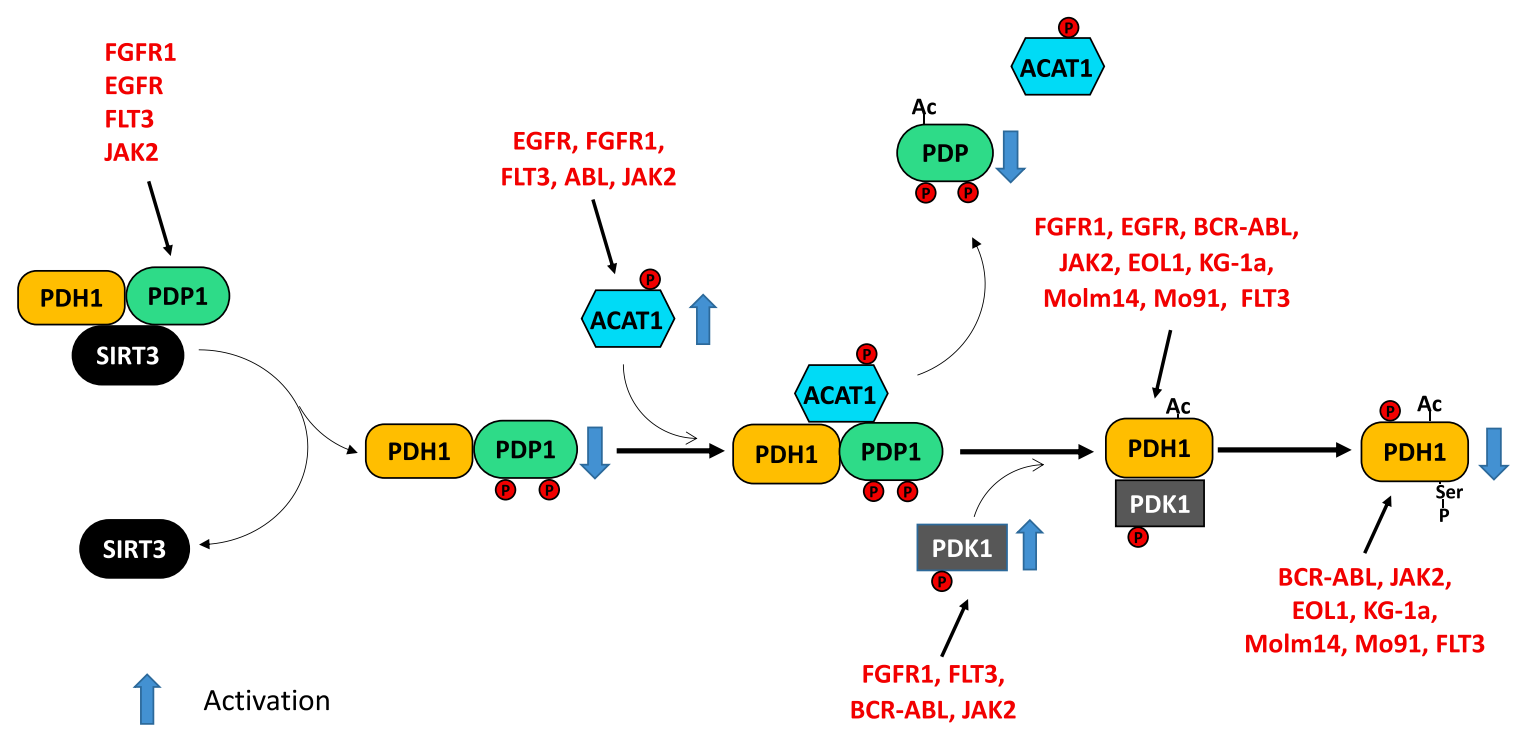

Inhibition

Fig. 4. Control of PDC activity through phosphorylation on tyrosine residues. In cancer cells, PDP1 is phosphorylated on Y94 and Y301 by oncogenic TKs. Phosphorylation of Y94 causes PDP1 inhibition, while phosphorylation of Y301 favours dissociation of SIRT3 and recruitment of ACAT1 to the PDH-PDP complex. After binding to the PDH-PDP complex, ACAT1, which is also activated by phosphorylation on tyrosine residue, acetylates PDP1 on K202 residue, impairing its ability to interact with PDHA. Once dissociated by PDP1, PDH can assemble with PDHK, which phosphorylates PDH on a serine residue inactivating it. In addition, the activity of PDHK is modulated by phosphorylation on tyrosine residue: several oncogenic TKs target PDHK, thus enhancing its activity. In addition, PDH is inhibited by phosphorylation on a tyrosine residue. The name of oncogenic tyrosine kinases responsible for phosphorylation of metabolic enzymes is reported in red. (For interpretation of the references to color in this figure legend, the reader is referred to the web version of this article.)

PDHK1 phosphorylation on different tyrosine residues, thereby promoting kinase activation [81]. These findings suggest that tyrosine phosphorylation, together with serine phosphorylation [83], plays a crucial role in inhibiting PDC activity and hence in promoting the glycolytic pathway in proliferating cancer cells (Fig. 4).

\subsubsection{Isocitrate dehydrogenase (IDH)}

Mammalian cells express three different IDH isoforms, namely IDH1, IDH2, and IDH3. IDH1 is localized in the cytoplasm, while IDH2 and IDH3 isoforms are mitochondrial enzymes [84]. The canonical function of IDH1/2 is to convert isocitrate to $\alpha \mathrm{KG}$ and $\mathrm{NADP}^{+}$to $\mathrm{NADPH}$. While the activity of IDH1/2 is $\mathrm{NADP}^{+}$-dependent, the activity of IDH3 isoform is strictly dependent on $\mathrm{NAD}^{+}$[85]. However, recent studies demonstrated that, under hypoxic conditions, IDH1/2 can also play a significant role in the reductive carboxylation by converting $\alpha$ $\mathrm{KG}$, generated from glutamine, into citrate, which is then released from mitochondria and destined to refill fatty acid biosynthetic pathways. This reductive, glutamine-dependent pathway is the main source of citrate in growing cancer cells [86,87].

Interestingly, the two isoenzymes, IDH1 and IDH2, are differently phosphorylated on tyrosine, suggesting that oncogenic TKs can exert a differential control on these enzymes. Noteworthy, the analysis of specimens derived from patients affected by different tumor types further confirmed that IDH1 is highly phosphorylated in vivo and that tyrosine phosphorylation of IDH1 is essential to maintain tumorigenic potential and to sustain cancer cell proliferation [88]. Consistently, it has been reported that the phosphorylation of Y42 and Y391 residues enhances the activity of IDH1 [88], promoting the conversion of isocitrate into $\alpha$-KG, which is an important substrate of several transaminases and an essential co-substrate for the Jumonji C-domain-containing $\alpha \mathrm{KG}$-dependent histone demethylases [89].

Specifically, Chen and co-authors showed that IDH1 isoenzyme is a target of two groups of TKs: the first group, including EGFR, JAK2, ABL1, PDGFR, and FGFR1, phosphorylates the Y42 residue, while the second group, composed by FLT3 and Src, enhances the phosphorylation of the Y391 residue. Phosphorylation of Y42 residue promotes the conversion of IDH1 from the monomeric (inactive) into the dimeric (active) form, thereby inducing substrate binding, while the phosphorylation of Y391 residue favours the binding of coenzyme $\mathrm{NADP}^{+}$[88].

Mutations in the genes encoding for IDH1/2 occur in various types of malignancies, including the majority of malignant gliomas and acute myeloid leukemia [90,91]. These mutations confer to IDH1/2 the function to produce 2-hydroxyglutarate (2-HG) and therefore to regulate cancer epigenetic by competitively inhibiting $\alpha$ KG-dependent TET enzymes [92].

Interestingly, the regulatory mechanism described by Chen and collaborators is effective on both wild-type and mutant IDH1. Indeed, oncogenic TKs can phosphorylate IDH1 mutated forms, stimulating the synthesis of 2-HG [88]. In particular, it has been demonstrated that tyrosine phosphorylation can affect the activity of IDH1 mutants, promoting the NADPH-dependent reduction of $\alpha$-KG into 2-HG, a competitive inhibitor of $\alpha$-KG-dependent dioxygenases [93].

In a recent paper, Ward and co-workers showed that cancer cells harbouring Y139D mutation in IDH1 increase the production of 2-HG [94]. Noteworthy, substitution of tyrosine with negatively charged residues was widely used to mimic constitutive phosphorylation of a protein or an enzyme [95]. Thus, it is possible to speculate that phosphorylation on Y139 residue, which is located close to the IDH1 active site, could contribute to the regulation of its activity, favouring the accumulation of 2-HG and HIF-1 $\alpha$ stabilization [96].

Together, these findings suggest that tyrosine phosphorylation has a key role in modulating the activity of both wild-type and mutated forms of IDH1.

Even if IDH is the only Krebs cycle enzyme for which the effect of tyrosine phosphorylation has been investigated, data obtained from PhosphoSitePlus database show that almost all the enzymes participating to the Krebs cycle, including citrate synthase (CS), aconitate hydratase (ACO2), IDH, succinil-CoA ligase subunit $\beta$ (SUCLA2 $\beta$, succinate dehydrogenase (SDHA), fumarate hydratase (FH), malate 
dehydrogenase (MDH2), can be phosphorylated on tyrosine residues.

Among these enzymes, data focused on ACO2 are particularly interesting in the context of cancer cells. The reductive carboxylation of $\alpha$-KG leads to the synthesis of a large amount of isocitrate, which must be converted into citrate before the extrusion from mitochondria. Therefore, we can argue that cancer metabolic reprogramming requires the inhibition of aconitase, the enzyme which catalyses the conversion of citrate into isocitrate. Studies performed on specimens from patients with gastric cancer revealed that the expression of ACO2 is significantly lower in gastric cancer tissues compared with matched adjacent nontumor tissues and that lower ACO2 expression is related to a poor prognosis [97]. Moreover, a recent study conducted on breast cancer cells showed that ACO2 expression decreases glucose consumption and increases oxidative metabolism [98]. Based on these observations and on the evidence that ACO2 is frequently phosphorylated on a tyrosine residue, it is possible to hypothesize that oncogenic TKs phosphorylate this enzyme in order to inhibit its activity, thereby promoting the Warburg effect and cancer cells proliferation.

No information is available about the impact of tyrosine phosphorylation on the activity of other enzymes involved in the Krebs cycle. However, it is important to notice that some intermediates of the Krebs cycle can act as regulatory metabolites. $\alpha-\mathrm{KG}$ is a co-substrate of HIF-1 $\alpha$ prolyl-hydroxylase, which catalyses HIF-1 $\alpha$ degradation. When the activity of $\alpha$-ketoglutarate dehydrogenase is inhibited or downregulated, $\alpha$-KG can accumulate and can be converted into L-2-HG by LDHA and MDH1 or mitochondrial MDH2. In turn, L-2-HG inhibits $\mathrm{PDH}$, resulting in HIF-1 $\alpha$ stabilization and in the promotion of Warburg metabolism [99]. Similarly, inhibition of SDH promotes the accumulation of succinate, while inhibition of $\mathrm{FH}$ induces accumulation of fumarate, two well described inhibitors of PDH. Coherently, the inhibition of both SDH and FH favours the activation of HIF-1 $\alpha$, which in turn up-regulates metabolic genes that promote the Warburg metabolism [100]. This finding suggests that oncogenic TKs could control the activity of these enzymes, finally leading to an increase of the intracellular concentration of the so-called "oncometabolites" that promote cancer progression though different adaptations.

\subsection{Mitochondrial electron chain complexes}

It is becoming increasingly clear that cancer cells do not simply rely on aerobic glycolysis for their bioenergetic and biosynthetic needs, thus for many types of cancer an increase in oxidative metabolism has been described [101]. Moreover, growing evidence demonstrates that cancer stem cells and cancer cells that acquired drug resistance frequently display an increase in mitochondrial metabolism [102-104], suggesting that oxidative metabolism is correlated with a more aggressive phenotype in cancers. The importance of this metabolic modulation is demonstrated by the positive antitumoral effects of several different OXPHOS inhibitors [105], confirming, on one hand, the hypothesis reported above that mitochondria play a key role in favouring cancer cells proliferation and, on the other, suggesting that tumor metabolic reprogramming includes deep modifications in the activity of mitochondrial electron chain. Multiple findings underline that oncogenic TKs, participating in these modifications, phosphorylating and regulating the activity of mitochondrial electron chain complexes, as discussed in the following sections. In addition, PhosphoSitePlus database analysis revealed that all the mitochondrial complexes can be phosphorylated on tyrosine, indicating the importance of this post-transcriptional modification in modulating the balance between glycolytic and oxidative metabolism in cancer.

\subsubsection{NADH-dehydrogenase (Complex I)}

Complex I of the mitochondrial electron chain contains several Fe-S clusters and a flavoprotein FMN, that play a central role in the transfer of electrons to ubiquinone. NADH dehydrogenase acts as a proton pump driven by electron transfer and directs protons from the mitochondrial matrix to the intermembrane space of mitochondria, simultaneously converting $\mathrm{NADH}$ to $\mathrm{NAD}^{+}$.

Complex I activity preserves a correct $\mathrm{NAD}^{+} / \mathrm{NADH}$ ratio, thus controlling the synthesis of precursors such as aspartate, inducing HIF1 $\alpha$ signaling, and inhibiting mTORC1 signaling. In cancer cells, these Complex I-related events support cell proliferation, resistance to cell death, and promotion of metastasis [106]. Complex I has been found overexpressed in different types of cancer including metastatic colon carcinoma [107]. However, overexpression of Complex I could result in an excessive ROS production, with the consequent risk of inducing severe cell damage and impairing cell proliferation. Hence, it is reasonable to suppose that oncogenic TKs should be able to regulate the activity of this complex to meet the metabolic demand of cancer cells, avoiding the production of elevated ROS levels and cell death [108,109].

In this context, Src tyrosine kinase translocates into mitochondria, where it phosphorylates the NDUFB10 subunit of Complex I, increasing its activity and contributing to the maintenance of the mitochondrial membrane potential. It has been suggested that this phosphorylation could affect the assembly, activity and/or proton translocation ability of Complex I [108]. It is noteworthy that the correct assembly of Complex I prevents excessive production of ROS, protecting cells from oxidative damage. Thus, it is reasonable to hypothesize that Src-mediated NDUFB10 tyrosine phosphorylation minimises the production of ROS by Complex I [109]. Moreover, Cesaro and co-workers showed that other tyrosine residues of NDUFB10, namely Y55 and Y142, are phosphorylated, but the effect of their phosphorylation on Complex I activity remains to be clarified [110]. An independent study on glioblastoma cells revealed that the Y193 residue of NADH dehydrogenase [ubiquinone] flavoprotein 2 (NDUFV2) is phosphorylated by Src, and treatment with a Src inhibitor impairs $\mathrm{O}_{2}$ consumption and reduces ATP production. Similar effects have been observed by substituting Y193 with a residue that cannot be phosphorylated. Considering that NDUFV2 is located in the NADH binding region of Complex I, it has been proposed that Y193 phosphorylation could impair NADH dehydrogenase activity [111].

\subsubsection{Succinate-ubiquinone oxidoreductase (Complex II)}

Complex II is a four-subunits protein, containing a co-factor FAD, three $2 \mathrm{Fe}-2 \mathrm{~S}$ clusters, a succinate binding site and a ubiquinone binding site. Specifically, it is involved in the electron transfer from succinate to ubiquinone. Complex II plays an important role in supporting tumor progression as demonstrated by the successful results obtained by the application of Complex II inhibitors in the treatment of several types of cancer [112].

Genes encoding for different subunits of Complex II are mutated and epigenetically modulated in many types of cancer [113]. Moreover, recent studies demonstrated that the activity of Complex II can be also regulated by tyrosine phosphorylation $[114,115]$. Acin-Perez and coworkers reported that mitochondrial Fgr tyrosine kinase phosphorylates the Y604 residue of Complex II, increasing its activity and reducing the expression of Complex I [29]. Therefore, cells enhance $\mathrm{FADH}_{2}$ oxidation at the expense of NADH. Taking into account that NADH/FADH ratio is increased in glucose consuming cells and decreased in cells that metabolize fatty acids, it has been speculated that tyrosine phosphorylation of Complex II could promote the metabolic rewiring of cells under stress conditions, such as glucose deprivation [29].

\subsection{3. bc1 Complex (Complex III)}

Complex III is a multi-subunit complex that couples the electron transfer from the reduced ubiquinol to cytochrome $\mathrm{c}$ with proton transport from the mitochondrial matrix to mitochondrial intermembrane space.

A study conducted by Augereau and co-workers revealed that Complex III is tyrosine phosphorylated by Src kinase family members under either direct exposure of mitochondria to ATP in vitro or during in 
situ mitochondrial ATP production. However, to date, the role of this post-translational modification remains unknown [116].

\subsubsection{Cytochrome $c$ and cytochrome $c$ oxidase (Complex IV)}

Cytochrome $\mathrm{c}$ is a key modulator of mitochondrial activity. Its role consists in transferring electrons from the mitochondrial Complex III to the cytochrome c oxidase ( $\mathrm{CcO}$ ) complex, which in turn catalyses the reduction of molecular oxygen in two water molecules, using four electrons and four protons derived from the mitochondrial matrix. Moreover, cytochrome c can be released in the cytoplasm, where it is able to trigger apoptosis, leading to cell death [117]. Defective CcO has been related to increased glycolysis and to the induction of retrograde signaling which can induce tumor progression [118]. Independent studies evidenced that $\mathrm{CcO}$ is phosphorylated on several tyrosine residues by different TKs. Immobilized metal affinity chromatography (IMAC) coupled with mass spectrometry experiments revealed that two tyrosine residues of cytochrome c, namely Y48 and Y97, are phosphorylated by a yet unknown TK [119]. Kinetic analyses, performed on Y48 phosphorylated cytochrome c, showed that phosphorylation is detrimental for cytochrome $\mathrm{c}$ activity, decreasing the respiration rate by $50 \%$ [119]. Moreover, Pecina and co-workers proved that the substitution of Y48 residue with a phospho-mimetic group results in the inhibition of the apoptotic process [120]. Conversely, phosphorylation of Y97 residue does not impair the respiration rate, suggesting a different role of this residue in regulating cytochrome c activity [119]. It has been demonstrated that Y97 phosphorylation decreases the affinity of cytochrome $\mathrm{c}$ for $\mathrm{CcO}$, thereby modulating either the flux of electrons through the mitochondrial electron chain and the ATP synthesis [121]. Furthermore, considering that Y97 residue is located in a hydrophobic region implicated in the binding of phospholipids, the phosphorylation of this tyrosine probably modulates the interaction of cytochrome $\mathrm{c}$ with cardiolipin [122]. Notably, Y97 residue is phosphorylated in both healthy heart and brain, but fully dephosphorylated during ischemic events. Thus, Y97 dephosphorylation could contribute, on one hand, to regulate electron flux through mitochondrial electron chain when higher amounts of ATP are required, and, on the other, to limit cardiolipin oxidation, thereby protecting cells from apoptosis [123].

Lee and co-workers demonstrated that, in cow liver, the Y304 residue of $\mathrm{CcO}$ subunit I is phosphorylated following the treatment with an activator of adenylate-cyclase or the stimulation with glucagon, suggesting that a upstream TK is activated by the cAMP pathway. CcO phosphorylation on Y304 results in a strong inhibition its activity, promotes the dimerization of subunit I, thereby converting $\mathrm{CcO}$ in an allosteric protein, and causes a decrease in the Vmax and an increase in the $\mathrm{Km}$ for cytochrome c. Together these results confirm that Y304 phosphorylation leads to CcO inactivation in the liver [124]. Moreover, Samavati and co-authors showed that also the signaling pathway activated by TNF- $\alpha$, one of the most important mediators of inflammation, causes the activation of a mitochondrial TK that phosphorylates $\mathrm{CcO}$ on Y304 residue of subunit I, leading to $\mathrm{CcO}$ inhibition [125]. Similar experiments enable to identify $\mathrm{Y} 11$ residue as an alternative phosphorylation site at CcO subunit IV-1. Y11 residue is located in the ATP/ ADP binding site of CcO. Despite no mutagenesis studies have been performed to identify the functional role of Y11, computer modelling analysis [126] suggested that Y11 phosphorylation could have a role in modulating the allosteric behaviour of $\mathrm{CcO}$, mainly due to the proximity of this residue to the binding site for ADP and ATP [127].

Moreover, in silico analysis revealed that Y113 residue, an additional phosphorylation site of $\mathrm{CcO}$ subunit II, could be the target of cSrc tyrosine kinase. However, no data are available about the effect of Y113 phosphorylation on CcO activity [128].

\subsubsection{ATP synthase}

Mitochondrial ATP synthase, also known as Complex V, consists of two subunits $\left(F_{o}\right.$ and $\left.F_{1}\right)$ and is located in the internal mitochondrial membrane. It is responsible for the ATP synthesis from ADP and $\mathrm{P}_{\mathrm{i}}$. In addition, the presence of the proton gradient across the mitochondrial membrane allows the release of the newly synthesized ATP from the enzyme.

The role of ATP synthase downregulation in reprogramming energy metabolism of cancer cells has been summarized in a recent review [129]. Following its downregulation, cells enhance glycolytic flux, while reducing mitochondrial ATP production. Interestingly, the downregulation of ATP synthase and the concomitant overexpression of its inhibitory factor 1 (IF1) have been described in a subset of carcinomas, when compared to normal tissues $[130,131]$. Besides the regulation through IF1, ATP synthase activity seems to be also regulated trough post-translational modifications such as phosphorylation. Preliminary experiments performed in vitro on mouse cortical neurons revealed that stimulation with PDGF leads to tyrosine phosphorylation of the $\delta$ subunit of ATP synthase [132].

Further studies performed on mouse embryonic fibroblasts (NIH3T3 cells) and mesangial kidney cells confirmed that ATP synthase tyrosine phosphorylation is not only a prerogative of neurons [133]. The authors identified Y75 as the residue that undergoes phosphorylation following stimulation with PDGF, even if a subsequent bioinformatic analysis identified several other consensus sequences that should be targeted by cellular kinases [133]. Considering that the $\delta$ subunit is fundamental for the regulation of the "F1 motor unit" of the ATP synthase enzyme, it is reasonable to hypothesize that tyrosine phosphorylation of this subunit contributes to modulate ATP synthesis [133]. More recently, a study conducted on ATP synthase in resting mitochondria showed that Y52 residue in the $\gamma$ subunit is phosphorylated under steady-state conditions. The functional role of Y52 phosphorylation remains to be elucidated. Y52 residue is located at the foot of the $\gamma$ subunit and near the $\delta$ subunit [134]. Based on this evidence, we can suppose that phosphorylation of tyrosine residues located in the foot of the $\gamma$ subunit could be essential to regulate the activity of the F1 complex and consequently the synthesis of ATP.

\subsubsection{Adenine nucleotide translocase (ANT)}

After the addition of a phosphate moiety to ADP, the neo-synthesized ATP is exported out of mitochondria using the ADP/ATP translocase. In particular, ANT transfers $\mathrm{ADP}^{3-}$ from the mitochondrial intermembrane space to the matrix, while transports $\mathrm{ATP}^{4-}$ outside the mitochondrial matrix. This transfer is favoured by the electrochemical and proton gradient across the mitochondrial membrane.

Among the four different ANT isoforms, the isoform 2 (ANT2) is directly related to the metabolic adaptation observed during tumoral development and ensures the great metabolic flexibility that characterizes cancer cells [135]. Interestingly, overexpression of ANT2 also leads to apoptosis inhibition, thus favouring cancer cell survival [135].

NNT is a transmembrane protein, showing a deep binding site for ATP and ADP, which includes at least three tyrosine residues, namely Y186, Y190, and Y194. Interestingly, it has been observed that Y190 and Y194 residues are both phosphorylated and the mutagenesis of these two residues impairs the ability of cells to grow on non-fermentable carbon sources. Taking into account the localization of these residues, it can be suggested that their phosphorylation may alter the chemical-physical properties of the ADP/ATP binding site, thereby changing the specificity of the binding or the transport rate [116].

\subsection{Amino acid metabolism}

One hallmark of metabolic reprogramming in cancer cells is the reorganization of amino acid metabolism [136]. Cancer cells show a remarkable heterogeneity in terms of quantity and modality of amino acids utilization. Many cancer cells increase the consumption of glutamine, which is the most abundant amino acid in plasma and a source of nitrogen atoms, that in turn can be used to synthesize other amino acids or nucleotides $[137,138]$. Compelling evidence shows that glutamine-derived carbons can be used to refill the Krebs cycle $[139,140]$. 
For instance, glutamine-derived $\alpha$-KG can be used to synthesize citrate through the reductive pathway, to generate malate and pyruvate, or to produce oxaloacetate (OAA) and aspartate [141]. On the other hand, an increase in amino acid metabolism leads to a greater production of ammonium, a toxic by-product for mammalian cells. Nevertheless, it is surprising to note that many cancer cells display a reduced production of nitrogen waste since they redirect most of their nitrogen supplies toward biosynthetic pathways [142]. However, to date, the mechanisms by which cancer cells are able to maximize the use of nitrogen and carbon derived from amino acids remain to be fully explained.

Data obtained from the analysis of PhosphoSitePlus database reveal that many enzymes involved in amino acid metabolism are phosphorylated on tyrosine residues, including glutaminase (GLS), glutamate dehydrogenase 1 (GLUD1), glutamate dehydrogenase 2 (GLUD2), cytosolic aspartate aminotransferase (GOT1), mitochondrial aspartate aminotransferase (GOT2), alanine aminotransferase (GPT1), glutamine synthetase (GLUL) and asparagine synthetase (ASNS) (Supplementary Table S5). Moreover, also the enzymes of the urea cycle, including mitochondrial carbamoyl-phosphate synthase (CPS1), argininosuccinate synthase (ASS1), and arginase 1 (ARG1), result to be phosphorylated on tyrosine residues (Supplementary Table S6). Together, these findings suggest that oncogenic TKs exert strict control on amino acid metabolism, allowing cancer cells to rewire their metabolism depending on nutrient and oxygen availability.

\subsubsection{Glutamine metabolism}

Right after glucose, glutamine is the second most consumed metabolite in cancer cells [139]. Glutamine is a key substrate for tumor growth as demonstrated by the strong sensitivity to glutamine deprivation observed in the majority of human cancer cell lines [143]. Moreover, the importance of glutamine metabolism in cancer is underlined by the fact that different anticancer treatments targeting distinct enzymes of this pathway have been successfully developed [144].

Oncogenic TKs are able to modulate different aspects of glutamine metabolism. For instance, they are involved in increasing glutamine uptake, through the upregulation of specific mitochondrial transporters [145,146], and increasing the activity of GLS [147]. GLS hydrolyses glutamine to glutamate, which can be further converted into $\alpha$-KG by GLUD1, thereby feeding the Krebs cycle. This pathway is crucial for proliferating cells since it replenishes the Krebs cycle with carbon atoms that can be used to different scopes, including the synthesis of nucleotides, lipids, and NADPH [140]. In a recent paper, Huang and coworkers showed that, in the liver, the MET kinase is able to increase the activity of GLS through phosphorylation on tyrosine residues [147]. To date, no information is available about the position and the number of tyrosine residues involved in the regulation of GLS activity. Moreover, it is interesting to note that MET kinase targets also PDC, leading to its inhibition, thus simultaneously stimulating the Warburg effect and the glutaminolysis, thereby promoting biogenesis and liver cancer cell growth [147] (Fig. 3).

Under hypoxia, many cancer cells increase the consumption of glutamine to sustain lipogenesis, nucleotide synthesis, and cell proliferation $[148,149]$. Interestingly, it has been demonstrated that cells excrete overflowed nitrogen and carbon in the form of dihydroorotate, an intermediate of pyrimidine synthesis [150]. In this context, oncogenic TKs could coordinate the activity of enzymes involved in amino acids catabolism and nucleotide synthesis, thereby sustaining cancer cell growth and avoiding toxic effect due to ammonia accumulation [150]. We can speculate that oncogenic TKs could promote this phenomenon by controlling the activity of CPS and GOT isoforms, the enzymes involved in the synthesis of carbamoyl phosphate and aspartate, two key metabolites required for purine and pyrimidine synthesis. Under glutamine deprivation, different types of cancer enhance the synthesis of glutamine starting from $\alpha$-KG. For instance, it has been reported that human breast cancer cells are able to incorporate nitrogen atoms from ammonia through the reductive amination reaction, catalysed by glutamate dehydrogenase (GDH), which converts $\alpha$-KG into glutamate. Moreover, glutamate can be used to sustain the synthesis of other amino acids such as proline and aspartate [142], or converted into glutamine by the reaction catalysed by GULL [151]. The finding that in cancer cells this enzyme results to be phosphorylated on different tyrosine residues suggest that oncogenic TKs could modulate its activity, thereby supporting cell survival and proliferation also under the condition of glutamine deprivation.

\subsubsection{Aspartate metabolism}

Aspartate is one of the most important amino acids for cancer cells because of its contribution to nucleotides biosynthesis [152]. Cancer cells can synthesize aspartate through different pathways: a first possibility is by exploiting the activity of GOT1 (cytosolic form) and GOT2 (mitochondrial form), two transaminases which convert glutamate into $\alpha-\mathrm{KG}$ and OAA into aspartate [153]; a second one is by converting asparagine into aspartate, using the enzyme asparaginase. It is interesting to observe that, according to PhosphoSitePlus database, all these enzymes are phosphorylated on tyrosine residues leading us to suppose that their activity is controlled by oncogenic TKs. It is noteworthy that the increase in aspartate levels can be obtained also by interfering with the activity of ASS1, the enzyme that synthesizes argininosuccinate, from aspartate and citrulline. It has been reported that, in melanoma and osteosarcoma cells, the inhibition of ASS1 determines the upregulation of the CAD complex, designated at the pyrimidine synthesis, and the increase in tumor burden [154]. Data reported in this review show that ASS1 possesses many tyrosine residues that can be phosphorylated and on these bases, we can argue that oncogenic TKs could control also its activity, promoting cancer cell proliferation.

\subsubsection{Serine metabolism}

Beside ribose, nucleotides biosynthesis requires carbon atoms derived from different amino acids, including serine, glycine, and glutamine. The first one can be synthesized starting from the intermediate of the glycolysis 3-PG through the serine synthesis pathway (SSP) (Fig. 2). Several studies showed that, in many tumor types, the increased activity of SSP supports the synthesis of numerous molecules essential to maintain the high proliferation rate of cancer cells, such as nucleotides [155], amino acids, and phospholipids [156,157]. The increased flux through the SSP in cancer cells is generally supported by the amplification of PHGDH and by the 2-PG-induced activation of PHGDH $[158,159]$.

Moreover, patients with breast cancers overexpressing PHGDH have a poorer survival rate, indicating the importance of this pathway in tumorigenesis [157].

Interestingly, thanks to PosphoSitePlus database analysis we found that, although PHGDH and phosphoserine phosphatase (PSPH) are not targets of oncogenic TKs, both the phosphoserine aminotransferase (PSAT1, the second enzyme of the SSP) and the cytosolic isoform of serine hydroxymethyltransferase (SHMT1, the enzyme deputed to catalyse the reversible conversion of serine and tetrahydrofolate to glycine and 5,10-methylene tetrahydrofolate) are frequently phosphorylated on tyrosine residues in cancer cells (Supplementary Table S7). Even though no information is available to date, we could speculate that this phosphorylation may be functional to improve the activity of these enzymes, thereby enhancing the synthesis of serine and glycine. Moreover, it is interesting to observe that the mitochondrial isoform of SHMT (SHMT2) is not phosphorylated on tyrosine, suggesting that oncogenic TKs can selectively control the activity of the two different isoforms. Considering that mitochondrial serine metabolism produces a larger amount of NADH than its cytosolic counterpart, we can suppose that differential phosphorylation (and hence regulation) of both SHMT1 and SHMT2 isoforms could be functional, de facto, to sustain nucleotide biosynthetic pathways without impairing glycolytic flux [160]. 


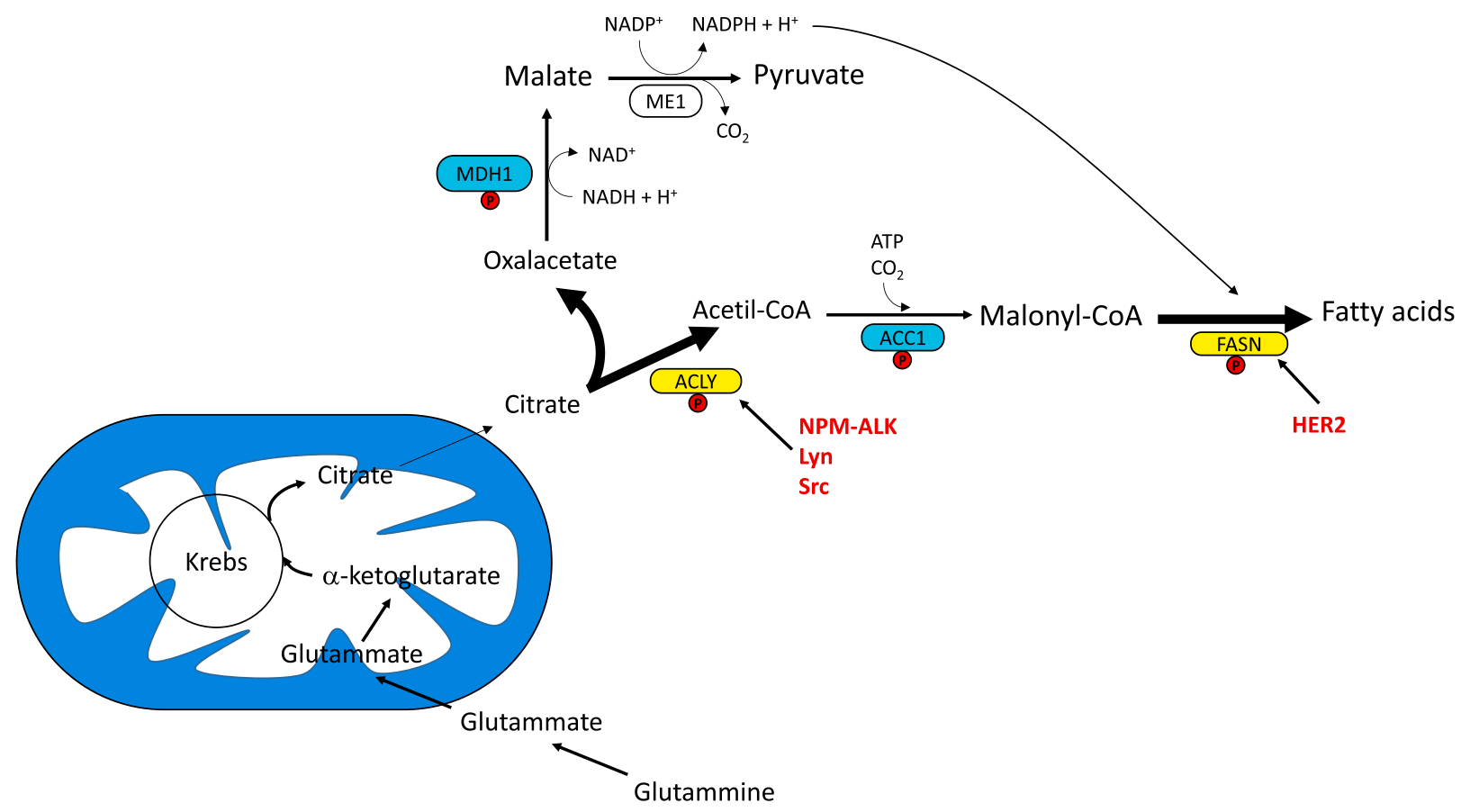

Fig. 5. Regulation of fatty acid synthesis by phosphorylation on tyrosine. The figure displays enzymes involved in fatty acid synthesis reporting in yellow those the activity of which is enhanced by phosphorylation on tyrosine residues (ACLY, FASN), and in light blue those for which the effects of the phosphorylation on tyrosine residues is still unknown. White boxes indicate not tyrosine phosphorylated enzymes. The name of oncogenic tyrosine kinases responsible for phosphorylation of metabolic enzymes is reported in red. The reactions that are activated by phosphorylation are indicated by thick ( $\longrightarrow$ ). (For interpretation of the references to color in this figure legend, the reader is referred to the web version of this article.)

\subsection{Lipid metabolism}

Lipids, together with glycerol, are the main constituents of cell membranes. In order to regulate their proliferation rate, cancer cells need to improve lipid biosynthesis, most of which is destined to support phospholipid production [161,162]. Moreover, cancer cells also enhance lipid uptake [163] and increase de novo lipid biosynthesis from glucose or glutamine [164], in order to meet their higher energetic demands.

The relevance of tyrosine phosphorylation on lipid metabolism has not yet received as much attention as for glucose or amino acid metabolism. However, some studies identified tyrosine phosphorylated sites on enzymes involved in lipid metabolism, suggesting new potential roles still not completely discovered [165]. All the following enzymes and their phosphorylation sites are mentioned in the Supplementary Table S8 and in the Fig. 5.

\subsubsection{ATP-citrate lyase (ACLY)}

The role of ACLY is to convert coenzyme-A and citrate to acetylCoA. ACLY is connected to different malignancies. In particular a correlation has been identified between ACLY overexpression and poor differentiation and prognosis in lung adenocarcinoma [166] and acute myeloid leukemia [167]. In highly proliferating cells, the activation of ACLY is essential to avoid the accumulation of citrate, which otherwise could inhibit PFK1, thereby blocking the glycolytic pathway [168]. Citrate produced by reductive carboxylation of glutamine is exported into the cytosol where it is converted into OAA and Acetyl-CoA, which is therefore redirected toward the fatty acid biosynthesis. This is possible thanks to the activity of oncogenic TKs that phosphorylate and activate both ACLY and ACAT1 enzymes [169,170].

Alongside the role of serine phosphorylation on ACLY activation, Basappa and co-workers identified ACLY as a possible substrate of the chimeric TK NPM-ALK. NPM-ALK is an oncogenic protein, typical of the anaplastic large cell lymphoma (ALCL), and it results from the recurrent translocations involving anaplastic lymphoma kinase (ALK) gene and the nucleophosmin (NPM) gene [169]. Indeed, phospho-proteomic analyses of three ALK-positive ALCL derived cell lines identified the Y682 residue of ACLY as a novel phosphorylation site of NPM-ALK. Expression of ACLY mutant Y682F or pharmacological inhibition of ALK resulted in decreased ACLY activity, as well as decreased lipid synthesis and increased fatty acid ß-oxidation when compared to ACLYWT. Moreover, ACLY-Y682F expression resulted in decreased cell proliferation, reduced clonogenic potential, and impaired tumor growth in xenograft mouse models. Thus, tyrosine phosphorylation of Y682 residue has been identified as a novel mechanism of ACLY regulation in ALCL pathogenesis [169]. More recently, the same authors described that Lyn and Src kinases directly phosphorylate ACLY on six and four tyrosine residues, respectively. Interestingly, the three ACLY tyrosine residues that are commonly phosphorylated bu Lyn and Src, namely Y682, Y252, Y227, are located in the catalytic domain, the citratebinding domain, and the ATP-binding domain, respectively, suggesting a key role for tyrosine phosphorylation in regulating the activation of the enzyme. Moreover, Lyn inhibition strongly reduces ACLY activity and impairs the growth of Acute Myeloid Leukemia patient derived cells [170].

\subsubsection{Fatty acid synthase (FASN)}

FASN is a central biosynthetic enzyme in the lipid metabolism, that plays a fundamental role in the de novo lipogenesis. Indeed, it is a homodimeric enzyme complex that, essentially, condenses acetyl-CoA and malonyl-CoA to produce long-chain fatty acids, using NADPH as cosubstrate. FASN has been found transcriptionally regulated by androgens, estradiol, and progesterone in hormonally responsive tumors. Moreover, FASN is overexpressed and hyperactivated in an aggressive subset of sex-steroid-related tumors, including breast carcinoma [171]. Recently, Menendez and co-authors identified that the anomalous lipogenic activity of tumor-associated FASN regulates the response of breast cancer cells to E2-stimulated ER $\alpha$ signaling. These authors demonstrated that FASN inhibition arrests E2-stimulated breast cancer cell proliferation and anchorage-independent colony formation through 
increased expression of the inhibitors p21WAF1/CIP1 and p27Kip1 and inactivation of AKT [171]. This evidence paves the way for promising therapeutic approaches for anticancer treatment based on FASN inhibitors. Indeed, small molecules inhibitors of FASN, including TVB2640, showed promising preclinical efficacy in several tumor types [172].

In 2010, Jin and co-authors demonstrated that in human SKBR3 breast cancer cells heregulin (HRG) activates the HER2 TK, which, in turn, interacts with and phosphorylates FASN. Tyrosine phosphorylation of FASN enhances enzyme activity, promoting de novo lipogenesis and cell invasion. It is interesting to notice that the treatment of SKBR3 cells with the HER2 inhibitor lapatinib halts both FASN phosphorylation and activity, thus inhibiting cancer cell invasion [173]. Interestingly, FASN phosphorylation on tyrosine residues suggests that oncogenic TKs, by regulating the activity of this enzyme, coordinate the production of fatty acid precursors with the synthesis of fatty acids.

\subsubsection{Acetyl-CoA acetyltransferase (ACAT1)}

ACAT1 is the mitochondrial isoform of Acetyl-CoA acetyltransferase. Besides, a cytosolic isoform has also been described, namely ACAT2. Specifically, ACAT1 is involved in the synthesis of acetoacetyl-CoA from two molecules of Acetyl-CoA during ketogenesis and ketolysis. In addition, it plays an important role in the isoleucine degradation pathway.

ACAT1 is commonly upregulated in different human cancer types such as leukemia, lung, head and neck and prostate cancers [174]. Fan and co-authors demonstrated that ACAT1 is active in its tetrameric form and that its tetramerization is facilitated by phosphorylation on Y407 residue, triggered by different TKs, such as EGFR, FGFR1, FLT3, ABL and JAK2 [175]. ACAT1 Y407 phosphorylation is crucial for cancer cell proliferation and tumor growth, favouring the metabolic switch from OXPHOS to aerobic glycolysis in tumor cells through the inhibition of PDC. As previously mentioned, PDP1 and PDHA1 are also inhibited by acetylation at K202 and K321 residues, respectively. Interestingly, as mentioned above, mitochondrial ACAT1 and SIRT3 are the upstream acetyltransferase and deacetylase of PDHA1 and PDP1, respectively [79]. In this scenario, oncogenic TKs are able to decrease PDC functionality and conversely increase the Warburg effect, thus promoting tumor growth, through the phosphorylation/activation of ACAT1. ACAT1, in turn, inhibits PDHA1 and PDP1 by means of lysine acetylation. In agreement, the Y407F ACAT1 mutant (or the pharmacological inhibition of ACAT1 with AH/arecoline, HBrn) leads to a decrease in ACAT1 activity [79]. These events correlate with increased PDHA1 activity (PDC flux rate) and OXPHOS, together with a concomitant decrease in glycolysis and lactate production, finally leading to the reduction of cell proliferation and tumor growth.

\subsubsection{Acyl-CoA synthetase}

Long-chain fatty acyl-CoAs are synthesized by the long-chain acylCoA synthetase (or ligase) isoforms. These isoforms are regulated independently and have different tissue expression patterns and subcellular locations. All the isozymes of this family, although differing in substrate specificity, subcellular localization, and tissue distribution, have a crucial role in lipid biosynthesis and fatty acid degradation, transforming free long-chain fatty acids into fatty acyl-CoA esters [176].

Twenty-five phosphorylation sites, including tyrosine residues, have been identified in long-chain-fatty-acid-CoA ligase isoform 1 (ACSL1) by mass spectrometry, in the liver and brown adipocytes, sustaining the importance of post-translational modifications in regulating ACSL1 activity [177].

ACSL4 is another isozyme of the long-chain fatty-acid-coenzyme A ligase family and it preferentially utilizes arachidonate as substrate [178]. It is interesting to notice that triple negative breast cancers (TNBCs) preferentially express ACSL isoforms with respect to receptor positive breast cancers (RPBC) [179]. In particular, the overexpression of ACLS4 in TNBCs leads to increased levels of arachidonyl-CoA and hence prostaglandins, thereby contributing to the more aggressive phenotype generally observed in TNBCs compared to RPBC [179]. Different phosphoproteomic studies identified tyrosine residues that can be phosphorylated on this protein, even if their functional significance is still not known. However, it has been established a correlation between ACSL4 protein expression and Src-homology region 2 domain-containing phosphatase-2 (SHP2) activity, although no involvement of the phosphorylation level of a specific tyrosine residue in ACSL4 has been highlighted. Indeed, overexpression of an active form of SHP2 increases ACSL4 protein levels in MA-10 Leydig steroidogenic cells, whereas knockdown of this phosphatase reduces ACSL4 mRNA and protein levels [180], inducing increased or decreased steroid production, respectively. Moreover, treatment with NSC87877, a specific inhibitor of the tyrosine phosphatase SHP2, causes a substantial decrease in the proliferation of MDA-MB-231 cells [180].

\section{Discussion}

Data reported in this review highlight that tyrosine phosphorylation is a widespread post-translational modification that regulates the activity of numerous key metabolic enzymes (Supplementary Tables S1-S8 and Fig. 6). The large number of records attributed to each single phosphorylation event leads us to exclude that tyrosine phosphorylation could be merely considered as a random event in the large scenario of cancer metabolism. Most importantly, among all the tyrosine residues identified through PosphoSitePlus database analysis, only a few of them were already described for their ability to regulate enzyme activity, while the function of other residues remains to be clarified (Supplementary Table S9). This finding suggests that the role of phosphorylation on tyrosine as a mechanism to control the activity of metabolic enzymes is still largely underestimated. In the last years, many TKs have been identified as molecular targets for inhibitory therapeutic strategies against tumors, either as monotherapy and in combination with chemotherapy, immunotherapy or other TK inhibitors (TKIs) [181-183]. TKIs generally inhibit the active site of tyrosine kinases, preventing the activation of downstream signaling pathways involved mainly in cell proliferation or angiogenesis. The relevance of this approach led to the approval, up to August 2019, of 43 receptor TKIs in oncology by the Food and Drug Administration (FDA) [184]. The widespread usage of TKIs in the clinic confirms the relevance of tyrosine phosphorylation in the progression of different tumor types. Besides the largely described effects on the inhibition of tumor growth and malignancy, TKIs often also promotes metabolic reprogramming. For instance, Imatinib Gefitinib, Erlotinib and Capmatinib impair glycolytic pathway and reactivate oxidative phosphorylation [185,186], while Sorafenib promotes mitochondrial dysfunction to eradicate cancer cells [187] However, to our knowledge, TKIs specifically targeting metabolic enzymes still remains to be identified. Therefore, the aforementioned relevance of tyrosine phosphorylation on metabolic enzymes and the efficacy of drugs targeting their activity [4], might pave the way for the development of therapeutics that specifically hinder the tyrosine phosphorylation of these enzymes for clinical use.

\subsection{Impact of tyrosine phosphorylation on the activity of metabolic enzymes}

The analyses of the data reported in the previous chapters revealed that tyrosine phosphorylation often acts as a "reversible switch", being able to modulate, directly or indirectly, the enzyme activity. For instance, tyrosine phosphorylation affects the association/dissociation of multimeric enzyme subunits or the interaction of the enzymes with other regulatory proteins, changes the affinity of the enzymes for their substrates and co-factors, and modulates their catalytic rate. Regarding the enzymes harbouring multiple phosphorylation sites, the prediction of the overall impact of multiple phosphorylation events on the enzyme 


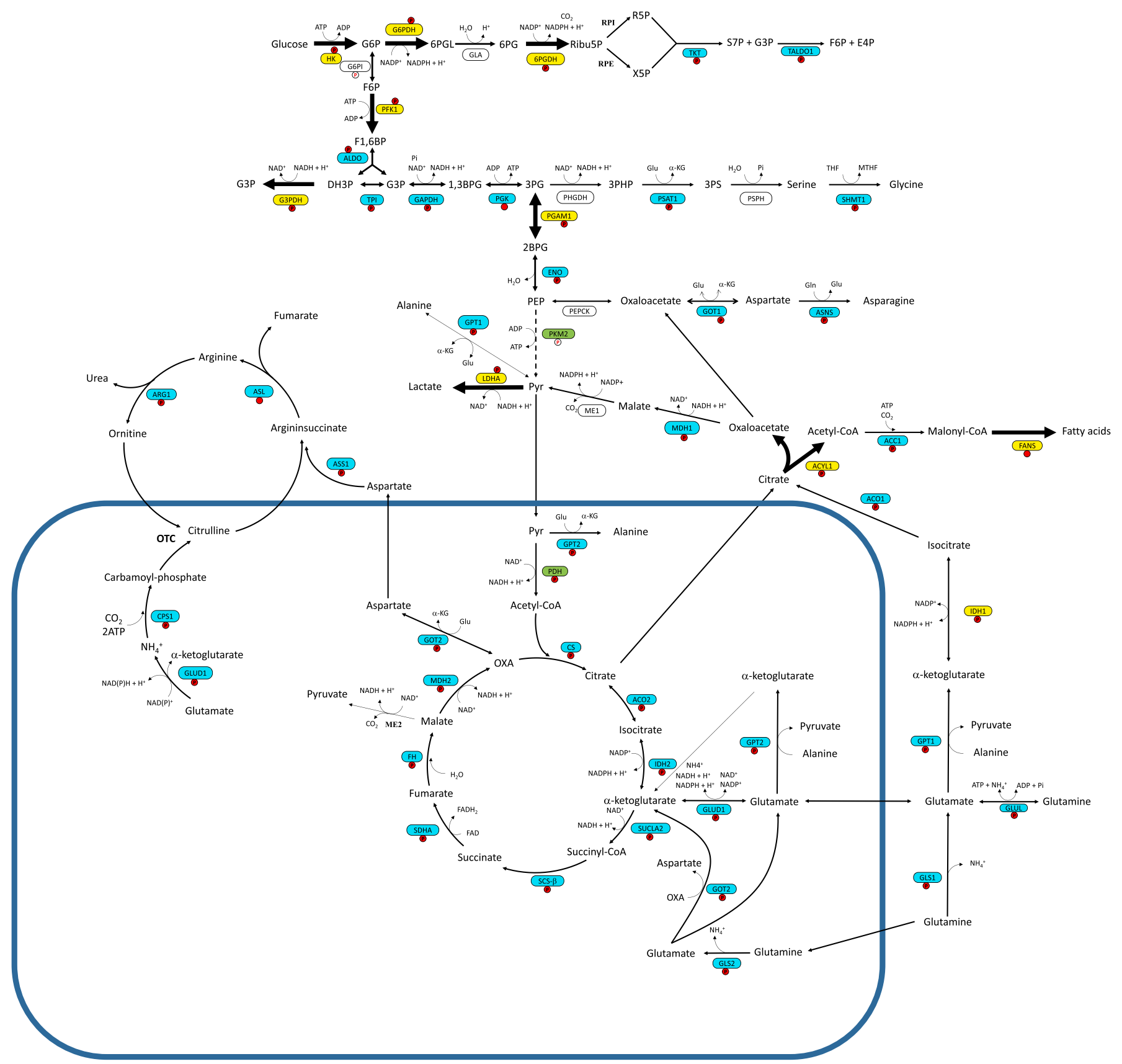

Fig. 6. Impact of tyrosine phosphorylation on metabolism-related enzymes. Overview of all the data obtained from PhosphoSitePlus database analysis representing the spread of tyrosine phosphorylation among metabolic enzymes, including those participating in glycolysis, PPP, Krebs cycle, oxidative phosphorylation, fatty acid metabolism, amino acid metabolism, and urea cycle. The figure shows glycolytic enzymes the activity of which is positively (yellow) or negatively (green) influenced by phosphorylation on tyrosine residues. The reactions that are activated or inhibited by phosphorylation are indicated by thick $(\longrightarrow$ ) and dashed arrows (- $\rightarrow$ ), respectively. Light blue boxes indicate the enzymes for which phosphorylation on tyrosine residues has been demonstrated, but whose effects on enzyme activity are not yet known. White boxes indicate not tyrosine phosphorylated enzymes. The name of oncogenic tyrosine kinases responsible for phosphorylation of metabolic enzymes is reported in red. (For interpretation of the references to color in this figure legend, the reader is referred to the web version of this article.)

activity is further complicated by the fact that the effect of the phosphorylation on each site could synergize with, or counteract, the effects due to the concomitant modification of other sites. Probably, these peculiar enzymes could act as "data integration hubs", being targets of different oncogenic TKs, activated by various incoming stimuli at the same time [24]. Multiple phosphorylation events will therefore result in a specific response representing the integration of the effects of the phosphorylation at all the different sites: this phenomenon could allow cancer cells to find the most appropriate energy resource even in the presence of divergent signals [188].

\subsection{The galaxy of tyrosine-phosphorylated metabolic enzymes}

Fig. 6 resumes all the data obtained from PhosphoSitePlus database analysis, showing that the number of metabolic enzymes phosphorylated on tyrosine is much larger than that described up to now and includes enzymes participating in glycolysis (Supplementary Table S1), PPP (Supplementary Table S2), Krebs cycle (Supplementary Table S4), OXPHOS, amino acid metabolism (Supplementary Tables S5 and S7) and urea cycle (Supplementary Table S6), and fatty acid metabolism (Supplementary Table S8). Although in most of the cases the effects of 
this post-translational modification on the enzyme activity are still unknown, its great spreading suggests that it should represent a key mechanism by which oncogenic TKs can control cancer metabolism. Altogether, this information is extremely interesting because, for the first time, reveals the true spread of tyrosine phosphorylation among metabolic enzymes.

\section{Conclusions}

Phosphorylation on tyrosine is a reversible, short-life phenomenon that involves key metabolic enzymes. We may suppose that it represents a formidable mechanism to modulate mammalian cell metabolism. Moreover, this post-translational modification can be used either to generate local and transitory events, including confined fluctuations of metabolic intermediates and long-term effects, such as a shift toward a more glycolytic or respiratory metabolism, depending on the incoming stimuli, the environmental conditions, the availability of nutrients and oxygen levels.

Despite the evident importance of this post-translational modification in establishing the metabolic profile of cancer cells, to date many issues still need to be clarified. First of all, the identification of the tyrosine phosphatases and kinases involved in this phenomenon is lacking for several reported enzymes. Another missing point is a more detailed characterization of the specific residues phosphorylated on the metabolic enzymes. Altogether, a better molecular understanding of these aspects may reveal new possible targets for the development of therapeutic strategies directed against the specific metabolism of cancer cells. Moreover, although data reported strongly support the key role of tyrosine phosphorylation in modulating cancer cell metabolism, the majority of them are the result of in vitro experimental settings, underlining the necessity of confirming the obtained data in models closer to clinical conditions.

Altogether, informations reported in this review provide, for the first time, an exhaustive picture of the extension of tyrosine phosphorylation among metabolic enzymes, highlighting its potential impact in regulating energy metabolism of cancer cells and encouraging future investigations of these aspects.

Supplementary data to this article can be found online at https:// doi.org/10.1016/j.bbcan.2020.188442.

\section{Funding sources}

This work was supported by Associazione Italiana Ricerca sul Cancro (AIRC) (project code: 19515) for the project "Assaying tumor metabolic deregulation in live cells", by University of Florence (Fondo ex-60\%), and by AIRC fellowship to Erica Pranzini (project code: 24132) for the project "Metabolic adaptations driving epigenetics of 5Fluorouracil-resistant colon cancer: the role of one carbon metabolism".

\section{Author contributions}

MLT, EP, EP, GR, PP: wrote the manuscript. PP: planned overall concepts.

\section{Declaration of Competing Interest}

The authors declare that they have no conflict of interest.

\section{References}

[1] O. Warburg, F. Wind, E. Negelein, The metabolism of tumors in the body, J. Gen. Physiol. 8 (6) (1927) 519-530.

[2] M.G. Vander Heiden, L.C. Cantley, C.B. Thompson, Understanding the Warburg effect: the metabolic requirements of cell proliferation, Science 324 (5930) (2009) 1029-1033.

[3] E. Gaude, C. Frezza, Defects in mitochondrial metabolism and cancer, Cancer Metab. 2 (2014) 10.
[4] P.E. Porporato, N. Filigheddu, J.M.B. Pedro, G. Kroemer, L. Galluzzi, Mitochondrial metabolism and cancer, Cell Res. 28 (3) (2018) 265-280.

[5] L.R. Cavalli, M. Varella-Garcia, B.C. Liang, Diminished tumorigenic phenotype after depletion of mitochondrial DNA, Cell Growth Differ. 8 (11) (1997) 1189-1198.

[6] P.S. Ward, C.B. Thompson, Metabolic reprogramming: a cancer hallmark even warburg did not anticipate, Cancer Cell 21 (3) (2012) 297-308.

[7] R.A. Cairns, I.S. Harris, T.W. Mak, Regulation of cancer cell metabolism, Nat. Rev. Cancer 11 (2) (2011) 85-95.

[8] A. Nagarajan, P. Malvi, N. Wajapeyee, Oncogene-directed alterations in cancer cell metabolism, Trends Cancer 2 (7) (2016) 365-377.

[9] P. Blume-Jensen, T. Hunter, Oncogenic kinase signalling, Nature 411 (6835) (2001) 355-365.

[10] M.K. Paul, A.K. Mukhopadhyay, Tyrosine kinase - role and significance in cancer, Int. J. Med. Sci. 1 (2) (2004) 101-115.

[11] Y. Ding, Z. Liu, S. Desai, Y. Zhao, H. Liu, L.K. Pannell, H. Yi, E.R. Wright, L.B. Owen, W. Dean-Colomb, O. Fodstad, J. Lu, S.P. LeDoux, G.L. Wilson, M. Tan, Receptor tyrosine kinase ErbB2 translocates into mitochondria and regulates cellular metabolism, Nat. Commun. 3 (2012) 1271.

[12] T. Yamaoka, S. Kusumoto, K. Ando, M. Ohba, T. Ohmori, Receptor tyrosine kinasetargeted cancer therapy, Int. J. Mol. Sci. 19 (11) (2018).

[13] D. Fabbro, S. Ruetz, E. Buchdunger, S.W. Cowan-Jacob, G. Fendrich, J. Liebetanz, J. Mestan, T. O'Reilly, P. Traxler, B. Chaudhuri, H. Fretz, J. Zimmermann, T. Meyer, G. Caravatti, P. Furet, P.W. Manley, Protein kinases as targets for anticancer agents: from inhibitors to useful drugs, Pharmacol. Ther. 93 (2-3) (2002) 79-98.

[14] R. Roskoski, A historical overview of protein kinases and their targeted small molecule inhibitors, Pharmacol. Res. 100 (2015) 1-23.

[15] A. Bettaieb, J. Bakke, N. Nagata, K. Matsuo, Y. Xi, S. Liu, D. AbouBechara, R. Melhem, K. Stanhope, B. Cummings, J. Graham, A. Bremer, S. Zhang, C.A. Lyssiotis, Z.Y. Zhang, L.C. Cantley, P.J. Havel, F.G. Haj, Protein tyrosine phosphatase 1B regulates pyruvate kinase M2 tyrosine phosphorylation, J. Biol. Chem. 288 (24) (2013) 17360-17371.

[16] G. Lori, T. Gamberi, P. Paoli, A. Caselli, E. Pranzini, R. Marzocchini, A. Modesti, G. Raugei, LMW-PTP modulates glucose metabolism in cancer cells, Biochim. Biophys. Acta Gen. Subj. 1862 (12) (2018) 2533-2544.

[17] S.J. Humphrey, D.E. James, M. Mann, Protein phosphorylation: a major switch mechanism for metabolic regulation, Trends Endocrinol. Metab. 26 (12) (2015) 676-687.

[18] J. Fan, T. Hitosugi, T.W. Chung, J. Xie, Q. Ge, T.L. Gu, R.D. Polakiewicz, G.Z. Chen, T.J. Boggon, S. Lonial, F.R. Khuri, S. Kang, J. Chen, Tyrosine phosphorylation of lactate dehydrogenase $\mathrm{A}$ is important for $\mathrm{NADH} / \mathrm{NAD}(+)$ redox homeostasis in cancer cells, Mol. Cell. Biol. 31 (24) (2011) 4938-4950.

[19] T. Hitosugi, L. Zhou, J. Fan, S. Elf, L. Zhang, J. Xie, Y. Wang, T.L. Gu, M. Alečković, G. LeRoy, Y. Kang, H.B. Kang, J.H. Seo, C. Shan, P. Jin, W. Gong, S. Lonial, M.L. Arellano, H.J. Khoury, G.Z. Chen, D.M. Shin, F.R. Khuri, T.J. Boggon, S. Kang, C. He, J. Chen, Tyr26 phosphorylation of PGAM1 provides a metabolic advantage to tumours by stabilizing the active conformation, Nat. Commun. 4 (2013) 1790.

[20] J. Zhang, S. Wang, B. Jiang, L. Huang, Z. Ji, X. Li, H. Zhou, A. Han, A. Chen, Y. Wu, H. Ma, W. Zhao, Q. Zhao, C. Xie, X. Sun, Y. Zhou, H. Huang, M. Suleman, F. Lin, L. Zhou, F. Tian, M. Jin, Y. Cai, N. Zhang, Q. Li, c-Src phosphorylation and activation of hexokinase promotes tumorigenesis and metastasis, Nat. Commun. 8 (2017) 13732

[21] T. Hitosugi, S. Kang, M.G. Vander Heiden, T.W. Chung, S. Elf, K. Lythgoe, S. Dong, S. Lonial, X. Wang, G.Z. Chen, J. Xie, T.L. Gu, R.D. Polakiewicz, J.L. Roesel, T.J. Boggon, F.R. Khuri, D.G. Gilliland, L.C. Cantley, J. Kaufman, J. Chen, Tyrosine phosphorylation inhibits PKM2 to promote the Warburg effect and tumor growth, Sci. Signal 2 (97) (2009) ra73.

[22] J.H. Lee, R. Liu, J. Li, Y. Wang, L. Tan, X.J. Li, X. Qian, C. Zhang, Y. Xia, D. Xu, W. Guo, Z. Ding, L. Du, Y. Zheng, Q. Chen, P.L. Lorenzi, G.B. Mills, T. Jiang, Z. Lu, EGFR-phosphorylated platelet isoform of phosphofructokinase 1 promotes PI3K activation, Mol. Cell. 70 (2) (2018) 197-210 (e7).

[23] S. Pan, C.J. World, C.J. Kovacs, B.C. Berk, Glucose 6-phosphate dehydrogenase is regulated through c-Src-mediated tyrosine phosphorylation in endothelial cells, Arterioscler. Thromb. Vasc. Biol. 29 (6) (2009) 895-901.

[24] P. Cohen, The regulation of protein function by multisite phosphorylation-a 25 year update, Trends Biochem. Sci. 25 (12) (2000) 596-601.

[25] P.V. Hornbeck, B. Zhang, B. Murray, J.M. Kornhauser, V. Latham, E. Skrzypek, PhosphoSitePlus, 2014: mutations, PTMs and recalibrations, Nucleic Acids Res 43 (Database issue) (2015) D512-D520.

[26] M.V. Liberti, J.W. Locasale, The Warburg effect: how does it benefit cancer cells? Trends Biochem. Sci. 41 (3) (2016) 211-218.

[27] S. Xu, A. Catapang, D. Braas, L. Stiles, H.M. Doh, J.T. Lee, T.G. Graeber, R. Damoiseaux, O. Shirihai, H.R. Herschman, A precision therapeutic strategy for hexokinase 1-null, hexokinase 2-positive cancers, Cancer Metab. 6 (2018) 7.

[28] A. Hennipman, J. Smits, B. van Oirschot, J.C. van Houwelingen, G. Rijksen, J.P. Neyt, J.A. Van Unnik, G.E. Staal, Glycolytic enzymes in breast cancer, benign breast disease and normal breast tissue, Tumour Biol. 8 (5) (1987) 251-263.

[29] R. Acín-Pérez, I. Carrascoso, F. Baixauli, M. Roche-Molina, A. Latorre-Pellicer, P. Fernández-Silva, M. Mittelbrunn, F. Sanchez-Madrid, A. Pérez-Martos, C.A. Lowell, G. Manfredi, J.A. Enríquez, ROS-triggered phosphorylation of complex II by Fgr kinase regulates cellular adaptation to fuel use, Cell Metab. 19 (6) (2014) 1020-1033.

[30] A. Yalcin, S. Telang, B. Clem, J. Chesney, Regulation of glucose metabolism by 6 phosphofructo-2-kinase/fructose-2,6-bisphosphatases in cancer, Exp. Mol. Pathol. 86 (3) (2009) 174-179. 
[31] J.H. Lee, F. Shao, J. Ling, S. Lu, R. Liu, L. Du, J.W. Chung, S.S. Koh, S.H. Leem, J. Shao, D. Xing, Z. An, Z. Lu, Phosphofructokinase 1 platelet isoform promotes $\beta$ Catenin transactivation for tumor development, Front. Oncol. 10 (2020) 211.

[32] W.H. Lee, J.S. Choi, M.R. Byun, K.T. Koo, S. Shin, S.K. Lee, Y.J. Surh, Functional inactivation of triosephosphate isomerase through phosphorylation during etoposide-induced apoptosis in HeLa cells: potential role of Cdk2, Toxicology 278 (2) (2010) 224-228.

[33] F. Malentacchi, R. Marzocchini, S. Gelmini, C. Orlando, M. Serio, G. Ramponi, G. Raugei, Up-regulated expression of low molecular weight protein tyrosine phosphatases in different human cancers, Biochem. Biophys. Res. Commun. 334 (3) (2005) 875-883.

[34] C. Tristan, N. Shahani, T.W. Sedlak, A. Sawa, The diverse functions of GAPDH: views from different subcellular compartments, Cell. Signal. 23 (2) (2011) 317-323.

[35] G. Butera, N. Mullappilly, F. Masetto, M. Palmieri, M.T. Scupoli, R. Pacchiana, M. Donadelli, Regulation of Autophagy by Nuclear GAPDH and Its Aggregates in Cancer and Neurodegenerative Disorders, Int. J. Mol. Sci. 20 (9) (2019).

[36] E.J. Tisdale, Glyceraldehyde-3-phosphate dehydrogenase is phosphorylated by protein kinase Ciota/lambda and plays a role in microtubule dynamics in the early secretory pathway, J. Biol. Chem. 277 (5) (2002) 3334-3341.

[37] E.J. Tisdale, C.R. Artalejo, A GAPDH mutant defective in Src-dependent tyrosine phosphorylation impedes Rab2-mediated events, Traffic 8 (6) (2007) 733-741.

[38] M. Phadke, N. Krynetskaia, A. Mishra, C. Barrero, S. Merali, S.A. Gothe, E. Krynetskiy, Disruption of NAD $(+)$ binding site in glyceraldehyde 3-phosphate dehydrogenase affects its intranuclear interactions, World J. Biol. Chem. 6 (4) (2015) 366-378.

[39] Y. He, Y. Luo, D. Zhang, X. Wang, P. Zhang, H. Li, S. Ejaz, S. Liang, PGK1-mediated cancer progression and drug resistance, Am. J. Cancer Res. 9 (11) (2019) 2280-2302.

[40] P. Shetty, T. Velusamy, Y.P. Bhandary, M.C. Liu, S. Shetty, Urokinase receptor expression involves tyrosine phosphorylation of phosphoglycerate kinase, Mol. Cell. Biochem. 335 (1-2) (2010) 235-247.

[41] A. Laurenzana, A. Chillà, C. Luciani, S. Peppicelli, A. Biagioni, F. Bianchini, E. Tenedini, E. Torre, A. Mocali, L. Calorini, F. Margheri, G. Fibbi, M. Del Rosso, UPA/uPAR system activation drives a glycolytic phenotype in melanoma cells, Int. J. Cancer 141 (6) (2017) 1190-1200.

[42] F. Sharif, A. Rasul, A. Ashraf, G. Hussain, T. Younis, I. Sarfraz, M.A. Chaudhry, S.A. Bukhari, X.Y. Ji, Z. Selamoglu, M. Ali, Phosphoglycerate mutase 1 in cancer: a promising target for diagnosis and therapy, IUBMB Life 71 (10) (2019) 1418-1427.

[43] T. Hitosugi, L. Zhou, S. Elf, J. Fan, H.B. Kang, J.H. Seo, C. Shan, Q. Dai, L. Zhang, J. Xie, T.L. Gu, P. Jin, M. Alečković, G. LeRoy, Y. Kang, J.A. Sudderth, R.J. DeBerardinis, C.H. Luan, G.Z. Chen, S. Muller, D.M. Shin, T.K. Owonikoko, S. Lonial, M.L. Arellano, H.J. Khoury, F.R. Khuri, B.H. Lee, K. Ye, T.J. Boggon, S. Kang, C. He, J. Chen, Phosphoglycerate mutase 1 coordinates glycolysis and biosynthesis to promote tumor growth, Cancer Cell 22 (5) (2012) 585-600.

[44] R.C. Oslund, X. Su, M. Haugbro, J.M. Kee, M. Esposito, Y. David, B. Wang, E. Ge, D.H. Perlman, Y. Kang, T.W. Muir, J.D. Rabinowitz, Bisphosphoglycerate mutase controls serine pathway flux via 3-phosphoglycerate, Nat. Chem. Biol. 13 (10) (2017) 1081-1087.

[45] P. Zhan, S. Zhao, H. Yan, C. Yin, Y. Xiao, Y. Wang, R. Ni, W. Chen, G. Wei, P. Zhang, $\alpha$-enolase promotes tumorigenesis and metastasis via regulating AMPK/ mTOR pathway in colorectal cancer, Mol. Carcinog. 56 (5) (2017) 1427-1437.

[46] X. Qian, W. Xu, J. Xu, Q. Shi, J. Li, Y. Weng, Z. Jiang, L. Feng, X. Wang, J. Zhou, H. Jin, Enolase 1 stimulates glycolysis to promote chemoresistance in gastric cancer, Oncotarget 8 (29) (2017) 47691-47708.

[47] J.A. Cooper, F.S. Esch, S.S. Taylor, T. Hunter, Phosphorylation sites in enolase and lactate dehydrogenase utilized by tyrosine protein kinases in vivo and in vitro, J. Biol. Chem. 259 (12) (1984) 7835-7841.

[48] K. Zahra, T. Dey, S.P. Mishra Ashish, U. Pandey, Pyruvate Kinase M2 and cancer: the role of PKM2 in promoting tumorigenesis, Front. Oncol. 10 (2020) 159.

[49] Q. Su, S. Luo, Q. Tan, J. Deng, S. Zhou, M. Peng, T. Tao, X. Yang, The role of pyruvate kinase M2 in anticancer therapeutic treatments, Oncol. Lett. 18 (6) (2019) 5663-5672.

[50] S.O. Lim, C.W. Li, W. Xia, H.H. Lee, S.S. Chang, J. Shen, J.L. Hsu, D. Raftery, D. Djukovic, H. Gu, W.C. Chang, H.L. Wang, M.L. Chen, L. Huo, C.H. Chen, Y. Wu, A. Sahin, S.M. Hanash, G.N. Hortobagyi, M.C. Hung, EGFR signaling enhances aerobic glycolysis in triple-negative breast cancer cells to promote tumor growth and immune escape, Cancer Res. 76 (5) (2016) 1284-1296.

[51] P. Kalaiarasan, N. Subbarao, R.N. Bamezai, Molecular simulation of Tyr105 phosphorylated pyruvate kinase M2 to understand its structure and dynamics, J. Mol. Model. 20 (9) (2014) 2447.

[52] C.V. Dang, PKM2 tyrosine phosphorylation and glutamine metabolism signal a different view of the Warburg effect, Sci. Signal. 2 (97) (2009) pe75.

[53] A. Najafov, D.R. Alessi, Uncoupling the Warburg effect from cancer, Proc. Natl. Acad. Sci. U. S. A. 107 (45) (2010) 19135-19136.

[54] C.V. Dang, Rethinking the Warburg effect with Myc micromanaging glutamine metabolism, Cancer Res. 70 (3) (2010) 859-862.

[55] N.M. Grüning, D. Du, M.A. Keller, B.F. Luisi, M. Ralser, Inhibition of triosephosphate isomerase by phosphoenolpyruvate in the feedback-regulation of glycolysis, Open Biol. 4 (2014) 130232.

[56] G. Dong, Q. Mao, W. Xia, Y. Xu, J. Wang, L. Xu, F. Jiang, PKM2 and cancer: the function of PKM2 beyond glycolysis, Oncol. Lett. 11 (3) (2016) 1980-1986.

[57] Y. Feng, Y. Xiong, T. Qiao, X. Li, L. Jia, Y. Han, Lactate dehydrogenase A: a key player in carcinogenesis and potential target in cancer therapy, Cancer Med. 7 (12) (2018) 6124-6136.
[58] L.R. Gray, S.C. Tompkins, E.B. Taylor, Regulation of pyruvate metabolism and human disease, Cell. Mol. Life Sci. 71 (14) (2014) 2577-2604.

[59] L. Jin, J. Chun, C. Pan, G.N. Alesi, D. Li, K.R. Magliocca, Y. Kang, Z.G. Chen, D.M. Shin, F.R. Khuri, J. Fan, S. Kang, Phosphorylation-mediated activation of LDHA promotes cancer cell invasion and tumour metastasis, Oncogene 36 (27) (2017) 3797-3806.

[60] A. Le, C.R. Cooper, A.M. Gouw, R. Dinavahi, A. Maitra, L.M. Deck, R.E. Royer, D.L. Vander Jagt, G.L. Semenza, C.V. Dang, Inhibition of lactate dehydrogenase A induces oxidative stress and inhibits tumor progression, Proc. Natl. Acad. Sci. U. S. A. 107 (5) (2010) 2037-2042.

[61] J. Liu, G. Chen, Z. Liu, S. Liu, Z. Cai, P. You, Y. Ke, L. Lai, Y. Huang, H. Gao, L. Zhao, H. Pelicano, P. Huang, W.L. McKeehan, C.L. Wu, C. Wang, W. Zhong, F. Wang, Aberrant FGFR tyrosine kinase signaling enhances the Warburg effect by reprogramming LDH isoform expression and activity in prostate cancer, Cancer Res. 78 (16) (2018) 4459-4470.

[62] K.G. de la Cruz-López, L.J. Castro-Muñoz, D.O. Reyes-Hernández, A. GarcíaCarrancá, J. Manzo-Merino, Lactate in the regulation of tumor microenvironment and therapeutic approaches, Front. Oncol. 9 (2019) 1143.

[63] D.M. Dawson, T.L. Goodfriend, N.O. Kaplan, Lactic dehydrogenases: functions of the two types rates of synthesis of the two major forms can be correlated with metabolic differentiation, Science 143 (3609) (1964) 929-933.

[64] A. Cheng, P. Zhang, B. Wang, D. Yang, X. Duan, Y. Jiang, T. Xu, J. Shi, C. Ding, G. Wu, Z. Sang, O. Wu, H. Wang, M. Wu, Z. Zhang, X. Pan, Y.Y. Pan, P. Gao, H. Zhang, C.Z. Zhou, J. Guo, Z. Yang, Aurora-A mediated phosphorylation of LDHB promotes glycolysis and tumor progression by relieving the substrate-inhibition effect, Nat. Commun. 10 (1) (2019) 5566.

[65] S. Li, J. Gao, X. Zhuang, C. Zhao, X. Hou, X. Xing, C. Chen, Q. Liu, S. Liu, Y. Luo, Cyclin G2 inhibits the Warburg effect and tumour progression by suppressing LDHA phosphorylation in glioma, Int. J. Biol. Sci. 15 (3) (2019) 544-555.

[66] K.C. Patra, N. Hay, The pentose phosphate pathway and cancer, Trends Biochem. Sci. 39 (8) (2014) 347-354.

[67] H.C. Yang, Y.H. Wu, W.C. Yen, H.Y. Liu, T.L. Hwang, A. Stern, D.T. Chiu, The redox role of G6PD in cell growth, cell death, and cancer, Cells 8 (9) (2019).

[68] R. Liu, W. Li, B. Tao, X. Wang, Z. Yang, Y. Zhang, C. Wang, H. Gao, J. Liang, W. Yang, Tyrosine phosphorylation activates 6-phosphogluconate dehydrogenase and promotes tumor growth and radiation resistance, Nat. Commun. 10 (1) (2019) 991.

[69] I.M. Xu, R.K. Lai, S.H. Lin, A.P. Tse, D.K. Chiu, H.Y. Koh, C.T. Law, C.M. Wong, Z. Cai, C.C. Wong, I.O. Ng, Transketolase counteracts oxidative stress to drive cancer development, Proc. Natl. Acad. Sci. U. S. A. 113 (6) (2016) E725-E734.

[70] M. Thapa, G. Dallmann, Role of coenzymes in cancer metabolism, Semin. Cell Dev. Biol. 98 (2020) 44-53.

[71] C.W. Tseng, W.H. Kuo, S.H. Chan, H.L. Chan, K.J. Chang, L.H. Wang, Transketolase regulates the metabolic switch to control breast cancer cell metastasis via the $\alpha$-Ketoglutarate signaling pathway, Cancer Res. 78 (11) (2018) 2799-2812.

[72] P.A. Gameiro, L.A. Laviolette, J.K. Kelleher, O. Iliopoulos, G. Stephanopoulos, Cofactor balance by nicotinamide nucleotide transhydrogenase (NNT) coordinates reductive carboxylation and glucose catabolism in the tricarboxylic acid (TCA) cycle, J. Biol. Chem. 288 (18) (2013) 12967-12977.

[73] H.Y. Ho, Y.T. Lin, G. Lin, P.R. Wu, M.L. Cheng, Nicotinamide nucleotide transhydrogenase (NNT) deficiency dysregulates mitochondrial retrograde signaling and impedes proliferation, Redox Biol. 12 (2017) 916-928.

[74] S. Li, Z. Zhuang, T. Wu, J.C. Lin, Z.X. Liu, L.F. Zhou, T. Dai, L. Lu, H.Q. Ju, Nicotinamide nucleotide transhydrogenase-mediated redox homeostasis promotes tumor growth and metastasis in gastric cancer, Redox Biol. 18 (2018) 246-255.

[75] K. Yaku, K. Okabe, K. Hikosaka, T. Nakagawa, NAD metabolism in cancer therapeutics, Front. Oncol. 8 (2018) 622

[76] A.A. Sauve, D.Y. Youn, Sirtuins: NAD (+)-dependent deacetylase mechanism and regulation, Curr. Opin. Chem. Biol. 16 (5-6) (2012) 535-543.

[77] N.J. German, M.C. Haigis, Sirtuins and the metabolic hurdles in cancer, Curr. Biol. 25 (13) (2015) R569-R583.

[78] K. Sajnani, F. Islam, R.A. Smith, V. Gopalan, A.K. Lam, Genetic alterations in Krebs cycle and its impact on cancer pathogenesis, Biochimie 135 (2017) 164-172.

[79] J. Fan, C. Shan, H.B. Kang, S. Elf, J. Xie, M. Tucker, T.L. Gu, M. Aguiar, S. Lonning, H. Chen, M. Mohammadi, L.M. Britton, B.A. Garcia, M. Alečković, Y. Kang, S. Kaluz, N. Devi, E.G. Van Meir, T. Hitosugi, J.H. Seo, S. Lonial, M. Gaddh, M. Arellano, H.J. Khoury, F.R. Khuri, T.J. Boggon, S. Kang, J. Chen, Tyr phosphorylation of PDP1 toggles recruitment between ACAT1 and SIRT3 to regulate the pyruvate dehydrogenase complex, Mol. Cell 53 (4) (2014) 534-548.

[80] C. Shan, H.B. Kang, S. Elf, J. Xie, T.L. Gu, M. Aguiar, S. Lonning, T. Hitosugi, T.W. Chung, M. Arellano, H.J. Khoury, D.M. Shin, F.R. Khuri, T.J. Boggon, J. Fan, Tyr-94 phosphorylation inhibits pyruvate dehydrogenase phosphatase 1 and promotes tumor growth, J. Biol. Chem. 289 (31) (2014) 21413-21422.

[81] J. Fan, H.B. Kang, C. Shan, S. Elf, R. Lin, J. Xie, T.L. Gu, M. Aguiar, S. Lonning, T.W. Chung, M. Arellano, H.J. Khoury, D.M. Shin, F.R. Khuri, T.J. Boggon, S. Kang, J. Chen, Tyr-301 phosphorylation inhibits pyruvate dehydrogenase by blocking substrate binding and promotes the Warburg effect, J. Biol. Chem. 289 (38) (2014) 26533-26541.

[82] T. Hitosugi, J. Fan, T.W. Chung, K. Lythgoe, X. Wang, J. Xie, Q. Ge, T.L. Gu, R.D. Polakiewicz, J.L. Roesel, G.Z. Chen, T.J. Boggon, S. Lonial, H. Fu, F.R. Khuri, S. Kang, J. Chen, Tyrosine phosphorylation of mitochondrial pyruvate dehydrogenase kinase 1 is important for cancer metabolism, Mol. Cell 44 (6) (2011) 864-877.

[83] E. Saunier, C. Benelli, S. Bortoli, The pyruvate dehydrogenase complex in cancer: an old metabolic gatekeeper regulated by new pathways and pharmacological 
agents, Int. J. Cancer 138 (4) (2016) 809-817.

[84] H. Al-Khallaf, Isocitrate dehydrogenases in physiology and cancer: biochemical and molecular insight, Cell Biosci. 7 (2017) 37.

[85] C. Zhang, L.M. Moore, X. Li, W.K. Yung, W. Zhang, IDH1/2 mutations target a key hallmark of cancer by deregulating cellular metabolism in glioma, NeuroOncology 15 (9) (2013) 1114-1126.

[86] C.M. Metallo, P.A. Gameiro, E.L. Bell, K.R. Mattaini, J. Yang, K. Hiller, C.M. Jewell, Z.R. Johnson, D.J. Irvine, L. Guarente, J.K. Kelleher, M.G. Vander Heiden, O. Iliopoulos, G. Stephanopoulos, Reductive glutamine metabolism by IDH1 mediates lipogenesis under hypoxia, Nature 481 (7381) (2011) 380-384.

[87] S.M. Fendt, E.L. Bell, M.A. Keibler, B.A. Olenchock, J.R. Mayers, T.M. Wasylenko, N.I. Vokes, L. Guarente, M.G. Vander Heiden, G. Stephanopoulos, Reductive glutamine metabolism is a function of the $\alpha$-ketoglutarate to citrate ratio in cells, Nat. Commun. 4 (2013) 2236.

[88] D. Chen, S. Xia, M. Wang, R. Lin, Y. Li, H. Mao, M. Aguiar, C.A. Famulare, A.H. Shih, C.W. Brennan, X. Gao, Y. Pan, S. Liu, J. Fan, L. Jin, L. Song, A. Zhou, J. Mukherjee, R.O. Pieper, A. Mishra, J. Peng, M. Arellano, W.G. Blum, S. Lonial, T.J. Boggon, R.L. Levine, J. Chen, Mutant and wild-type isocitrate dehydrogenase 1 share enhancing mechanisms involving distinct tyrosine kinase cascades in cancer, Cancer Discov. 9 (6) (2019) 756-777.

[89] S. Inoue, F. Lemonnier, T.W. Mak, Roles of IDH1/2 and TET2 mutations in myeloid disorders, Int. J. Hematol. 103 (6) (2016) 627-633.

[90] H. Yan, D.W. Parsons, G. Jin, R. McLendon, B.A. Rasheed, W. Yuan, I. Kos, I. Batinic-Haberle, S. Jones, G.J. Riggins, H. Friedman, A. Friedman, D. Reardon, J. Herndon, K.W. Kinzler, V.E. Velculescu, B. Vogelstein, D.D. Bigner, IDH1 and IDH2 mutations in gliomas, N. Engl. J. Med. 360 (8) (2009) 765-773.

[91] G. Montalban-Bravo, C.D. DiNardo, The role of IDH mutations in acute myeloid leukemia, Future Oncol. 14 (10) (2018) 979-993.

[92] L. Dang, D.W. White, S. Gross, B.D. Bennett, M.A. Bittinger, E.M. Driggers, V.R. Fantin, H.G. Jang, S. Jin, M.C. Keenan, K.M. Marks, R.M. Prins, P.S. Ward, K.E. Yen, L.M. Liau, J.D. Rabinowitz, L.C. Cantley, C.B. Thompson, M.G. Vander Heiden, S.M. Su, Cancer-associated IDH1 mutations produce 2-hydroxyglutarate, Nature 462 (7274) (2009) 739-744.

[93] R. Chowdhury, K.K. Yeoh, Y.M. Tian, L. Hillringhaus, E.A. Bagg, N.R. Rose, I.K. Leung, X.S. Li, E.C. Woon, M. Yang, M.A. McDonough, O.N. King, I.J. Clifton, R.J. Klose, T.D. Claridge, P.J. Ratcliffe, C.J. Schofield, A. Kawamura, The oncometabolite 2-hydroxyglutarate inhibits histone lysine demethylases, EMBO Rep. 12 (5) (2011) 463-469.

[94] P.S. Ward, J.R. Cross, C. Lu, O. Weigert, O. Abel-Wahab, R.L. Levine, D.M. Weinstock, K.A. Sharp, C.B. Thompson, Identification of additional IDH mutations associated with oncometabolite R(-)-2-hydroxyglutarate production, Oncogene 31 (19) (2012) 2491-2498.

[95] L.A. Kane, M.J. Youngman, R.E. Jensen, J.E. Van Eyk, Phosphorylation of the F(1) F(o) ATP synthase beta subunit: functional and structural consequences assessed in a model system, Circ. Res. 106 (3) (2010) 504-513.

[96] G. Jin, Z.J. Reitman, I. Spasojevic, I. Batinic-Haberle, J. Yang, O. Schmidt-Kittler, D.D. Bigner, H. Yan, 2-hydroxyglutarate production, but not dominant negative function, is conferred by glioma-derived NADP-dependent isocitrate dehydrogenase mutations, PLoS One 6 (2) (2011) e16812.

[97] P. Wang, C. Mai, Y.L. Wei, J.J. Zhao, Y.M. Hu, Z.L. Zeng, J. Yang, W.H. Lu, R.H. Xu, P. Huang, Decreased expression of the mitochondrial metabolic enzyme aconitase (ACO2) is associated with poor prognosis in gastric cancer, Med. Oncol. 30 (2) (2013) 552.

[98] F. Ciccarone, L. Di Leo, G. Lazzarino, G. Maulucci, F. Di Giacinto, B. Tavazzi, M.R. Ciriolo, Aconitase 2 inhibits the proliferation of MCF-7 cells promoting mitochondrial oxidative metabolism and ROS/FoxO1-mediated autophagic response, Br. J. Cancer 122 (2) (2020) 182-193.

[99] W.M. Oldham, C.B. Clish, Y. Yang, J. Loscalzo, Hypoxia-mediated increases in L-2hydroxyglutarate coordinate the metabolic response to reductive stress, Cell Metab. 22 (2) (2015) 291-303.

[100] M.A. Selak, S.M. Armour, E.D. MacKenzie, H. Boulahbel, D.G. Watson, K.D. Mansfield, Y. Pan, M.C. Simon, C.B. Thompson, E. Gottlieb, Succinate links TCA cycle dysfunction to oncogenesis by inhibiting HIF-alpha prolyl hydroxylase, Cancer Cell 7 (1) (2005) 77-85.

[101] J. Zheng, Energy metabolism of cancer: glycolysis versus oxidative phosphorylation (review), Oncol. Lett. 4 (6) (2012) 1151-1157.

[102] P. Sancho, D. Barneda, C. Heeschen, Hallmarks of cancer stem cell metabolism, Br. J. Cancer 114 (12) (2016) 1305-1312.

[103] A. Viale, P. Pettazzoni, C.A. Lyssiotis, H. Ying, N. Sánchez, M. Marchesini, A. Carugo, T. Green, S. Seth, V. Giuliani, M. Kost-Alimova, F. Muller, S. Colla, L. Nezi, G. Genovese, A.K. Deem, A. Kapoor, W. Yao, E. Brunetto, Y. Kang, M. Yuan, J.M. Asara, Y.A. Wang, T.P. Heffernan, A.C. Kimmelman, H. Wang, J.B. Fleming, L.C. Cantley, R.A. DePinho, G.F. Draetta, Oncogene ablation-resistant pancreatic cancer cells depend on mitochondrial function, Nature 514 (7524) (2014) 628-632.

[104] D.S. Matassa, M.R. Amoroso, H. Lu, R. Avolio, D. Arzeni, C. Procaccini, D. Faicchia, F. Maddalena, V. Simeon, I. Agliarulo, E. Zanini, C. Mazzoccoli, C. Recchi, E. Stronach, G. Marone, H. Gabra, G. Matarese, M. Landriscina, F. Esposito, Oxidative metabolism drives inflammation-induced platinum resistance in human ovarian cancer, Cell Death Differ. 23 (9) (2016) 1542-1554.

[105] V. Sica, J.M. Bravo-San Pedro, G. Stoll, G. Kroemer, Oxidative phosphorylation as a potential therapeutic target for cancer therapy, Int. J. Cancer 146 (1) (2020) 10-17.

[106] F.A. Urra, F. Muñoz, A. Lovy, C. Cárdenas, The mitochondrial Complex(I)ty of cancer, Front. Oncol. 7 (2017) 118.

[107] J. Marquez, I. Kratchmarova, V. Akimov, F. Unda, G. Ibarretxe, A.S. Clerigué,
N. Osinalde, I. Badiola, NADH dehydrogenase complex I is overexpressed in incipient metastatic murine colon cancer cells, Oncol. Rep. 41 (2) (2019) 742-752.

[108] E. Hebert-Chatelain, C. Jose, N. Gutierrez Cortes, J.W. Dupuy, C. Rocher, J. Dachary-Prigent, T. Letellier, Preservation of NADH ubiquinone-oxidoreductase activity by Src kinase-mediated phosphorylation of NDUFB10, Biochim. Biophys. Acta 1817 (5) (2012) 718-725.

[109] E. Maranzana, G. Barbero, A.I. Falasca, G. Lenaz, M.L. Genova, Mitochondrial respiratory supercomplex association limits production of reactive oxygen species from complex I, Antioxid. Redox Signal. 19 (13) (2013) 1469-1480.

[110] L. Cesaro, M. Salvi, Mitochondrial tyrosine phosphoproteome: new insights from an up-to-date analysis, Biofactors 36 (6) (2010) 437-450.

[111] M. Ogura, J. Yamaki, M.K. Homma, Y. Homma, Mitochondrial c-Src regulates cell survival through phosphorylation of respiratory chain components, Biochem. J. 447 (2) (2012) 281-289.

[112] K. Kluckova, A. Bezawork-Geleta, J. Rohlena, L. Dong, J. Neuzil, Mitochondrial complex II, a novel target for anti-cancer agents, Biochim. Biophys. Acta 1827 (5) (2013) 552-564.

[113] A. Bezawork-Geleta, J. Rohlena, L. Dong, K. Pacak, J. Neuzil, Mitochondrial complex II: at the crossroads, Trends Biochem. Sci. 42 (4) (2017) 312-325.

[114] M. Salvi, N.A. Morrice, A.M. Brunati, A. Toninello, Identification of the flavoprotein of succinate dehydrogenase and aconitase as in vitro mitochondrial substrates of Fgr tyrosine kinase, FEBS Lett. 581 (29) (2007) 5579-5585.

[115] E. Tomitsuka, K. Kita, H. Esumi, Regulation of succinate-ubiquinone reductase and fumarate reductase activities in human complex II by phosphorylation of its flavoprotein subunit, Proc. Jpn. Acad. Ser. B Phys. Biol. Sci. 85 (7) (2009) 258-265.

[116] O. Augereau, S. Claverol, N. Boudes, M.J. Basurko, M. Bonneu, R. Rossignol, J.P. Mazat, T. Letellier, J. Dachary-Prigent, Identification of tyrosine-phosphorylated proteins of the mitochondrial oxidative phosphorylation machinery, Cell. Mol. Life Sci. 62 (13) (2005) 1478-1488.

[117] G. Kroemer, B. Dallaporta, M. Resche-Rigon, The mitochondrial death/life regulator in apoptosis and necrosis, Annu. Rev. Physiol. 60 (1998) 619-642.

[118] D.W. Dong, S. Srinivasan, M. Guha, N.G. Avadhani, Defects in cytochrome c oxidase expression induce a metabolic shift to glycolysis and carcinogenesis, Genom. Data 6 (2015) 99-107.

[119] H. Yu, I. Lee, A.R. Salomon, K. Yu, M. Hüttemann, Mammalian liver cytochrome c is tyrosine-48 phosphorylated in vivo, inhibiting mitochondrial respiration, Biochim. Biophys. Acta 1777 (7-8) (2008) 1066-1071.

[120] P. Pecina, G.G. Borisenko, N.A. Belikova, Y.Y. Tyurina, A. Pecinova, I. Lee, A.K. Samhan-Arias, K. Przyklenk, V.E. Kagan, M. Hüttemann, Phosphomimetic substitution of cytochrome $\mathrm{C}$ tyrosine 48 decreases respiration and binding to cardiolipin and abolishes ability to trigger downstream caspase activation, Biochemistry 49 (31) (2010) 6705-6714.

[121] A. Guerra-Castellano, A. Díaz-Quintana, G. Pérez-Mejías, C.A. Elena-Real, K. González-Arzola, S.M. García-Mauriño, M.A. De la Rosa, I. Díaz-Moreno, Oxidative stress is tightly regulated by cytochrome, Proc. Natl. Acad. Sci. U. S. A. 115 (31) (2018) 7955-7960.

[122] M. Rytömaa, P.K. Kinnunen, Evidence for two distinct acidic phospholipid-binding sites in cytochrome c, J. Biol. Chem. 269 (3) (1994) 1770-1774.

[123] H.A. Kalpage, V. Bazylianska, M.A. Recanati, A. Fite, J. Liu, J. Wan, N. Mantena, M.H. Malek, I. Podgorski, E.I. Heath, A. Vaishnav, B.F. Edwards, L.I. Grossman, T.H. Sanderson, I. Lee, M. Hüttemann, Tissue-specific regulation of cytochrome c by post-translational modifications: respiration, the mitochondrial membrane potential, ROS, and apoptosis, FASEB J. 33 (2) (2019) 1540-1553.

[124] I. Lee, A.R. Salomon, S. Ficarro, I. Mathes, F. Lottspeich, L.I. Grossman, M. Hüttemann, cAMP-dependent tyrosine phosphorylation of subunit I inhibits cytochrome c oxidase activity, J. Biol. Chem. 280 (7) (2005) 6094-6100.

[125] L. Samavati, I. Lee, I. Mathes, F. Lottspeich, M. Hüttemann, Tumor necrosis factor alpha inhibits oxidative phosphorylation through tyrosine phosphorylation at subunit I of cytochrome c oxidase, J. Biol. Chem. 283 (30) (2008) 21134-21144.

[126] M. Hüttemann, B. Kadenbach, L.I. Grossman, Mammalian subunit IV isoforms of cytochrome c oxidase, Gene 267 (1) (2001) 111-123.

[127] I. Lee, A.R. Salomon, K. Yu, L. Samavati, P. Pecina, A. Pecinova, M. Hüttemann, Isolation of regulatory-competent, phosphorylated cytochrome C oxidase, Methods Enzymol. 457 (2009) 193-210.

[128] M. Hüttemann, I. Lee, A. Pecinova, P. Pecina, K. Przyklenk, J.W. Doan, Regulation of oxidative phosphorylation, the mitochondrial membrane potential, and their role in human disease, J. Bioenerg. Biomembr. 40 (5) (2008) 445-456.

[129] P.B. Esparza-Moltó, J.M. Cuezva, The role of mitochondrial H, Front. Oncol. 8 (2018) 53.

[130] L. Sánchez-Cenizo, L. Formentini, M. Aldea, A.D. Ortega, P. García-Huerta, M. Sánchez-Aragó, J.M. Cuezva, Up-regulation of the ATPase inhibitory factor 1 (IF1) of the mitochondrial H+-ATP synthase in human tumors mediates the metabolic shift of cancer cells to a Warburg phenotype, J. Biol. Chem. 285 (33) (2010) 25308-25313.

[131] L. García-Ledo, C. Nuevo-Tapioles, C. Cuevas-Martín, I. Martínez-Reyes, B. Soldevilla, L. González-Llorente, J.M. Cuezva, Overexpression of the ATPase inhibitory factor 1 favors a non-metastatic phenotype in breast cancer, Front. Oncol. 7 (2017) 69.

[132] F.X. Zhang, W. Pan, J.B. Hutchins, Phosphorylation of F1F0 ATPase delta-subunit is regulated by platelet-derived growth factor in mouse cortical neurons in vitro, J. Neurochem. 65 (6) (1995) 2812-2815.

[133] Y.H. Ko, W. Pan, C. Inoue, P.L. Pedersen, Signal transduction to mitochondrial ATP synthase: evidence that PDGF-dependent phosphorylation of the delta-subunit occurs in several cell lines, involves tyrosine, and is modulated by lysophosphatidic acid, Mitochondrion 1 (4) (2002) 339-348.

[134] F. Di Pancrazio, E. Bisetto, V. Alverdi, I. Mavelli, G. Esposito, G. Lippe, Differential 
steady-state tyrosine phosphorylation of two oligomeric forms of mitochondrial F0F1ATPsynthase: a structural proteomic analysis, Proteomics 6 (3) (2006) 921-926.

[135] A. Chevrollier, D. Loiseau, P. Reynier, G. Stepien, Adenine nucleotide translocase 2 is a key mitochondrial protein in cancer metabolism, Biochim. Biophys. Acta 1807 (6) (2011) 562-567.

[136] L. Vettore, R.L. Westbrook, D.A. Tennant, New aspects of amino acid metabolism in cancer, Br. J. Cancer 122 (2) (2020) 150-156.

[137] A.M. Hosios, V.C. Hecht, L.V. Danai, M.O. Johnson, J.C. Rathmell, M.L. Steinhauser, S.R. Manalis, M.G. Vander Heiden, Amino acids rather than glucose account for the majority of cell mass in proliferating mammalian cells, Dev. Cell 36 (5) (2016) 540-549.

[138] A.J. Bott, S. Maimouni, W.X. Zong, The pleiotropic effects of glutamine metabolism in cancer, Cancers (Basel) 11 (6) (2019).

[139] J. Zhang, N.N. Pavlova, C.B. Thompson, Cancer cell metabolism: the essential role of the nonessential amino acid, glutamine, EMBO J. 36 (10) (2017) 1302-1315.

[140] R.J. DeBerardinis, A. Mancuso, E. Daikhin, I. Nissim, M. Yudkoff, S. Wehrli, C.B. Thompson, Beyond aerobic glycolysis: transformed cells can engage in glutamine metabolism that exceeds the requirement for protein and nucleotide synthesis, Proc. Natl. Acad. Sci. U. S. A. 104 (49) (2007) 19345-19350.

[141] T. Li, A. Le, Glutamine metabolism in cancer, Adv. Exp. Med. Biol. 1063 (2018) 13-32.

[142] J.B. Spinelli, H. Yoon, A.E. Ringel, S. Jeanfavre, C.B. Clish, M.C. Haigis, Metabolic recycling of ammonia via glutamate dehydrogenase supports breast cancer biomass, Science 358 (6365) (2017) 941-946.

[143] D.R. Wise, C.B. Thompson, Glutamine addiction: a new therapeutic target in cancer, Trends Biochem. Sci. 35 (8) (2010) 427-433.

[144] Y.K. Choi, K.G. Park, Targeting glutamine metabolism for cancer treatment, Biomol. Ther. (Seoul) 26 (1) (2018) 19-28.

[145] P. Gao, I. Tchernyshyov, T.C. Chang, Y.S. Lee, K. Kita, T. Ochi, K.I. Zeller, A.M. De Marzo, J.E. Van Eyk, J.T. Mendell, C.V. Dang, c-Myc suppression of miR-23a/b enhances mitochondrial glutaminase expression and glutamine metabolism, Nature 458 (7239) (2009) 762-765.

[146] D.R. Wise, R.J. DeBerardinis, A. Mancuso, N. Sayed, X.Y. Zhang, H.K. Pfeiffer, I. Nissim, E. Daikhin, M. Yudkoff, S.B. McMahon, C.B. Thompson, Myc regulates a transcriptional program that stimulates mitochondrial glutaminolysis and leads to glutamine addiction, Proc. Natl. Acad. Sci. U. S. A. 105 (48) (2008) 18782-18787.

[147] X. Huang, G. Gan, X. Wang, T. Xu, W. Xie, The HGF-MET axis coordinates liver cancer metabolism and autophagy for chemotherapeutic resistance, Autophagy 15 (7) (2019) 1258-1279.

[148] Z.F. Jiang, M. Wang, J.L. Xu, Y.J. Ning, Hypoxia promotes mitochondrial glutamine metabolism through HIF1 $\alpha$-GDH pathway in human lung cancer cells, Biochem. Biophys. Res. Commun. 483 (1) (2017) 32-38.

[149] R.C. Sun, N.C. Denko, Hypoxic regulation of glutamine metabolism through HIF1 and SIAH2 supports lipid synthesis that is necessary for tumor growth, Cell Metab. 19 (2) (2014) 285-292.

[150] Y. Wang, C. Bai, Y. Ruan, M. Liu, Q. Chu, L. Qiu, C. Yang, B. Li, Coordinative metabolism of glutamine carbon and nitrogen in proliferating cancer cells under hypoxia, Nat. Commun. 10 (1) (2019) 201.

[151] C.L. Collins, M. Wasa, W.W. Souba, S.F. Abcouwer, Regulation of glutamine synthetase in human breast carcinoma cells and experimental tumors, Surgery 122 (2) (1997) 451-463 discussion 463-4.

[152] L.B. Sullivan, D.Y. Gui, A.M. Hosios, L.N. Bush, E. Freinkman, M.G. Vander Heiden, Supporting aspartate biosynthesis is an essential function of respiration in proliferating cells, Cell 162 (3) (2015) 552-563.

[153] H.F. Alkan, J.G. Bogner-Strauss, Maintaining cytosolic aspartate levels is a major function of the TCA cycle in proliferating cells, Mol. Cell Oncol. 6 (5) (2019) e1536843.

[154] S.C. Nagamani, A. Erez, A metabolic link between the urea cycle and cancer cell proliferation, Mol. Cell Oncol. 3 (2) (2016) e1127314.

[155] M.A. Reid, A.E. Allen, S. Liu, M.V. Liberti, P. Liu, X. Liu, Z. Dai, X. Gao, Q. Wang, Y. Liu, L. Lai, J.W. Locasale, Serine synthesis through PHGDH coordinates nucleotide levels by maintaining central carbon metabolism, Nat. Commun. 9 (1) (2018) 5442.

[156] K.R. Mattaini, M.R. Sullivan, M.G. Vander Heiden, The importance of serine metabolism in cancer, J. Cell Biol. 214 (3) (2016) 249-257.

[157] M. Yang, K.H. Vousden, Serine and one-carbon metabolism in cancer, Nat. Rev. Cancer 16 (10) (2016) 650-662.

[158] J.W. Locasale, A.R. Grassian, T. Melman, C.A. Lyssiotis, K.R. Mattaini, A.J. Bass, G. Heffron, C.M. Metallo, T. Muranen, H. Sharfi, A.T. Sasaki, D. Anastasiou, E. Mullarky, N.I. Vokes, M. Sasaki, R. Beroukhim, G. Stephanopoulos, A.H. Ligon, M. Meyerson, A.L. Richardson, L. Chin, G. Wagner, J.M. Asara, J.S. Brugge, L.C. Cantley, M.G. Vander Heiden, Phosphoglycerate dehydrogenase diverts glycolytic flux and contributes to oncogenesis, Nat. Genet. 43 (9) (2011) 869-874.

[159] R. Possemato, K.M. Marks, Y.D. Shaul, M.E. Pacold, D. Kim, K. Birsoy, S. Sethumadhavan, H.K. Woo, H.G. Jang, A.K. Jha, W.W. Chen, F.G. Barrett, N. Stransky, Z.Y. Tsun, G.S. Cowley, J. Barretina, N.Y. Kalaany, P.P. Hsu, K. Ottina, A.M. Chan, B. Yuan, L.A. Garraway, D.E. Root, M. Mino-Kenudson, E.F. Brachtel, E.M. Driggers, D.M. Sabatini, Functional genomics reveal that the serine synthesis pathway is essential in breast cancer, Nature 476 (7360) (2011) 346-350.

[160] G.S. Ducker, J.D. Rabinowitz, One-carbon metabolism in health and disease, Cell Metab. 25 (1) (2017) 27-42.

[161] C.R. Santos, A. Schulze, Lipid metabolism in cancer, FEBS J. 279 (15) (2012)
$2610-2623$

[162] H.N. Abramson, The lipogenesis pathway as a cancer target, J. Med. Chem. 54 (16) (2011) 5615-5638.

[163] J. Zhao, Z. Zhi, C. Wang, H. Xing, G. Song, X. Yu, Y. Zhu, X. Wang, X. Zhang, Y. Di, Exogenous lipids promote the growth of breast cancer cells via CD36, Oncol. Rep. 38 (4) (2017) 2105-2115.

[164] F. Röhrig, A. Schulze, The multifaceted roles of fatty acid synthesis in cancer, Nat. Rev. Cancer 16 (11) (2016) 732-749.

[165] M. Poliaková, D.M. Aebersold, Y. Zimmer, M. Medová, The relevance of tyrosine kinase inhibitors for global metabolic pathways in cancer, Mol. Cancer 17 (1) (2018) 27.

[166] T. Migita, T. Narita, K. Nomura, E. Miyagi, F. Inazuka, M. Matsuura, M. Ushijima, T. Mashima, H. Seimiya, Y. Satoh, S. Okumura, K. Nakagawa, Y. Ishikawa, ATP citrate lyase: activation and therapeutic implications in non-small cell lung cancer, Cancer Res. 68 (20) (2008) 8547-8554.

[167] P. Icard, Z. Wu, L. Fournel, A. Coquerel, H. Lincet, M. Alifano, ATP citrate lyase: a central metabolic enzyme in cancer, Cancer Lett. 471 (2020) 125-134.

[168] S. Vora, R. Oskam, G.E. Staal, Isoenzymes of phosphofructokinase in the rat. Demonstration of the three non-identical subunits by biochemical, immunochemical and kinetic studies, Biochem. J. 229 (2) (1985) 333-341.

[169] J. Basappa, M.A. ElAzzouny, D. Rolland, T. Velusamy, S. Hwang, V. Basrur, K. Conlon, L. Zhao, N.G. Bailey, C.F. Burant, K.S.J. Elenitoba-Johnson, M.S. Lim, NPM-ALK mediated tyrosine phosphorylation of ATP citrate lyase regulates lipid metabolism and promotes oncogenesis of anaplastic large cell lymphoma, ASH57th Annual Meeting and Exposition, 5-8 Dec 2015 Orlando.

[170] J. Basappa, M. Citir, Q. Zhang, H.Y. Wang, X. Liu, O. Melnikov, H. Yahya, F. Stein, R. Muller, A. Traynor-Kaplan, C. Schultz, M.A. Wasik, A. Ptasznik, ACLY is the novel signaling target of PIP, Heliyon 6 (5) (2020) e03910.

[171] J.A. Menendez, R. Lupu, Fatty acid synthase regulates estrogen receptor- $\alpha$ signaling in breast cancer cells, Oncogenesis 6 (2) (2017) e299.

[172] D. Buckley, G. Duke, T.S. Heuer, M. O'Farrell, A.S. Wagman, W. McCulloch, G. Kemble, Fatty acid synthase - modern tumor cell biology insights into a classical oncology target, Pharmacol. Ther. 177 (2017) 23-31.

[173] Q. Jin, L.X. Yuan, D. Boulbes, J.M. Baek, Y.N. Wang, D. Gomez-Cabello, D.H. Hawke, S.C. Yeung, M.H. Lee, G.N. Hortobagyi, M.C. Hung, F.J. Esteva, Fatty acid synthase phosphorylation: a novel therapeutic target in HER2-overexpressing breast cancer cells, Breast Cancer Res. 12 (6) (2010) R96.

[174] A. Goudarzi, The recent insights into the function of ACAT1: a possible anti-cancer therapeutic target, Life Sci. 116592 (2019).

[175] J. Fan, R. Lin, S. Xia, D. Chen, S.E. Elf, S. Liu, Y. Pan, H. Xu, Z. Qian, M. Wang, C. Shan, L. Zhou, Q.Y. Lei, Y. Li, H. Mao, B.H. Lee, J. Sudderth, R.J. DeBerardinis, G. Zhang, T. Owonikoko, M. Gaddh, M.L. Arellano, H.J. Khoury, F.R. Khuri, S. Kang, P.W. Doetsch, S. Lonial, T.J. Boggon, W.J. Curran, J. Chen, Tetrameric acetyl-CoA acetyltransferase 1 is important for tumor growth, Mol. Cell 64 (5) (2016) 859-874.

[176] Y. Tang, J. Zhou, S.C. Hooi, Y.M. Jiang, G.D. Lu, Fatty acid activation in carcinogenesis and cancer development: essential roles of long-chain acyl-CoA synthetases, Oncol. Lett. 16 (2) (2018) 1390-1396.

[177] J.L. Frahm, L.O. Li, T.J. Grevengoed, R.A. Coleman, Phosphorylation and acetylation of Acyl-CoA synthetase-I, J. Proteomics Bioinform. 4 (7) (2011) 129-137.

[178] T.J. Grevengoed, E.L. Klett, R.A. Coleman, Acyl-CoA metabolism and partitioning, Annu. Rev. Nutr. 34 (2014) 1-30.

[179] M.E. Monaco, Fatty acid metabolism in breast cancer subtypes, Oncotarget 8 (17) (2017) 29487-29500.

[180] M. Cooke, U. Orlando, P. Maloberti, E.J. Podestá, F. Cornejo Maciel, Tyrosine phosphatase SHP2 regulates the expression of acyl-CoA synthetase ACSL4, J. Lipid Res. 52 (11) (2011) 1936-1948.

[181] Z. Yang, K.Y. Tam, Combination strategies using EGFR-TKi in NSCLC Therapy: learning from the gap between Pre-clinical results and clinical outcomes, Int. J. Biol. Sci. 14 (2) (2018) 204-216.

[182] A.W. Tolcher, W. Peng, E. Calvo, Rational approaches for combination therapy strategies targeting the MAP kinase pathway in solid tumors, Mol. Cancer Ther. 17 (1) (2018) 3-16.

[183] E. Rassy, R. Flippot, L. Albiges, Tyrosine kinase inhibitors and immunotherapy combinations in renal cell carcinoma, Ther. Adv. Med. Oncol. 12 (2020) 1758835920907504.

[184] C. Pottier, M. Fresnais, M. Gilon, G. Jérusalem, R. Longuespée, N.E. Sounni, Tyrosine kinase inhibitors in cancer: breakthrough and challenges of targeted therapy, Cancers (Basel) 12 (3) (2020).

[185] V. De Rosa, F. Iommelli, M. Monti, R. Fonti, G. Votta, M.P. Stoppelli, S. Del Vecchio, Reversal of Warburg effect and reactivation of oxidative phosphorylation by differential inhibition of EGFR signaling pathways in non-small cell lung cancer, Clin. Cancer Res. 21 (22) (2015) 5110-5120.

[186] S. Gottschalk, N. Anderson, C. Hainz, S.G. Eckhardt, N.J. Serkova, Imatinib (STI571)-mediated changes in glucose metabolism in human leukemia BCR-ABLpositive cells, Clin. Cancer Res. 10 (19) (2004) 6661-6668.

[187] V. Tesori, A.C. Piscaglia, D. Samengo, M. Barba, C. Bernardini, R. Scatena, A. Pontoglio, L. Castellini, J.N. Spelbrink, G. Maulucci, M.A. Puglisi, G. Pani, A. Gasbarrini, The multikinase inhibitor Sorafenib enhances glycolysis and synergizes with glycolysis blockade for cancer cell killing, Sci. Rep. 5 (2015) 9149.

[188] T. Hitosugi, J. Chen, Post-translational modifications and the Warburg effect, Oncogene 33 (34) (2014) 4279-4285. 